\title{
The effectiveness of education or behavioral interventions on adherence to phosphate control in adults receiving hemodialysis: A systematic review
}

\author{
Molly Milazi 1, 2, 3 \\ Ann Bonner 1, 2, 3 \\ Clint Douglas 1,3
}

1School of Nursing, Queensland University of Technology (QUT)

${ }^{2}$ Renal Nursing Professorial Unit, Kidney Health Services, Royal Brisbane and Women's Hospital

${ }^{3}$ Centre for Evidence-Based Healthy Ageing: An Affiliate Centre of the Joanna Briggs Institute

Corresponding author:

Molly Milazi

Email: molly.milazi@hdr.qut.edu.au

\section{Executive summary}

\section{Background}

People with end stage kidney disease develop impaired excretion of phosphate. Hyperphosphatemia develops in end stage kidney disease as a result of the kidneys' reduced ability to excrete ingested phosphate load and is characterized by high bone turnover, increased musculoskeletal morbidity including bone pain and muscle weakness. Increased serum phosphate levels are also associated with cardiovascular disease and associated mortality. These effects are significant considering cardiovascular disease is the leading cause of death in end stage kidney disease, making phosphate control a crucial treatment goal.

\section{Objectives}

To determine the effectiveness of education or behavioral interventions on adherence to phosphate control in adults with end stage kidney disease receiving hemodialysis.

Inclusion criteria

\section{Types of participants}

Studies included adults over the age of 18 years, with end stage kidney disease undergoing hemodialysis, attending dialysis facilities regardless of frequency and duration of treatment sessions per week. Studies with participants receiving hemodiafiltration were excluded. 


\section{Types of intervention(s)/phenomena of interest}

All types of education and behavioral interventions aimed at improving adherence to dietary phosphate restriction, phosphate binder medication and hemodialysis.

\section{Types of studies}

Randomized controlled trials, non-randomized controlled trials, before and after and cohort studies.

\section{Outcomes}

Outcome measures included serum phosphate levels, patient knowledge and adherence to phosphate control methods, chronic kidney disease self-management behavior and perceived selfefficacy for chronic kidney disease related to phosphate control.

\section{Search strategy}

A search was conducted in CINAHL, MEDLINE, The Cochrane Library, EMBASE, Web of Science, PsycINFO and ProQuest Dissertations \& Theses Global to find published studies, between January 2005 and December 2015.

\section{Methodological quality}

Risk of bias was assessed by three reviewers prior to inclusion in the review using standardized critical appraisal instruments from the Joanna Briggs Institute Meta-Analysis of Statistics Assessment and Review Instrument (JBI-MAStARI).

\section{Data extraction}

Data were extracted using the standardized data extraction tool from JBI-MAStARI.

\section{Data synthesis}

Data were pooled using JBI software. Mean differences $(95 \% \mathrm{Cl})$ and effect size estimates were calculated for continuous outcomes. Meta-analysis using a random-effects model was performed for serum phosphate levels, and where the findings could not be pooled using meta-analysis, results were presented in a narrative form. Standard GRADE evidence assessment of outcomes is reported.

\section{Results}

A total of 18 studies were included in the review: seven studies focused on dietary phosphate, four studies focused on medications (phosphate binders), and six studies focused on dietary phosphate and medications. Only one study taught patients about diet, medications and hemodialysis to control phosphate. Sixteen studies showed significant improvements in phosphate levels. Meta-analysis of eight RCTs favoured educational or behavioral interventions over standard care for serum phosphate control, with a weighted mean reduction of $-0.23 \mathrm{mmol} / \mathrm{L}(95 \% \mathrm{Cl}-0.37,-0.08)$ in treatment groups. 


\section{Conclusions}

Overall, educational or behavioral interventions increase adherence to phosphate control. Studies in this systematic review revealed improved outcomes on serum phosphate levels, patient knowledge and adherence to phosphate control methods, chronic kidney disease self-management behavior and perceived self-efficacy for chronic kidney disease related to phosphate control. However, there is a lack of sufficient data on how some of the studies implemented their interventions suggesting further research is required. Successful strategies that improve and optimize long term adherence to phosphate control are still needed to be formulated.

\section{Implications for practice}

The evidence supports the effectiveness of education or behavioral interventions for improving phosphate control in adults receiving hemodialysis. However, some evidence suggests that education sessions given over a longer period (teaching that is repeated/reinforced) assists patients to sustain phosphate control adherence.

\section{Implications for research}

Further robust studies are required to inform practice as most studies focused on either dietary phosphate or phosphate binder medication. A bundled, patient-centered intervention could improve phosphate control by supporting adherence with dietary restriction, phosphate binding medications and dialysis.

\section{Keywords}

Adherence, end stage kidney disease (ESKD), chronic kidney disease (CKD), hemodialysis (HD), phosphate control, educational, behavioral, knowledge, self-management 
Table 1: Summary of findings

Summary of findings:

Education or behavioral interventions compared to standard care for adherence to phosphate control in adults receiving hemodialysis

Patient or population: Adults receiving hemodialysis

Intervention: Education or behavioral interventions

Comparison: Standard care

\begin{tabular}{|c|c|c|c|c|c|c|}
\hline \multirow[t]{2}{*}{ Outcome } & \multicolumn{3}{|c|}{ Number of participants } & \multirow[t]{2}{*}{ Mean Difference } & \multirow{2}{*}{$\begin{array}{l}\text { Test for overall } \\
\text { effect }\end{array}$} & \multirow{2}{*}{$\begin{array}{l}\text { Quality of the } \\
\text { evidence } \\
\text { (GRADE) }\end{array}$} \\
\hline & $\begin{array}{l}\text { Education or } \\
\text { behavioral } \\
\text { intervention }\end{array}$ & $\begin{array}{l}\text { Standard } \\
\text { care }\end{array}$ & Total & & & \\
\hline \multirow{2}{*}{$\begin{array}{l}\text { Serum } \\
\text { phosphate } \\
\text { level }\end{array}$} & \multirow[t]{2}{*}{$n=408$} & \multirow[t]{2}{*}{$n=382$} & \multirow{2}{*}{$\begin{array}{l}n=790 \\
\text { (8 RCTs) }\end{array}$} & $\mathrm{D}=-0.23 \mathrm{mmol} / \mathrm{L}$ & \multirow[t]{2}{*}{$Z=3.01, p=0.003$} & $\bigoplus \bigoplus \bigoplus$ \\
\hline & & & & $\begin{array}{l}95 \% \mathrm{Cl} \quad(-0.37,- \\
0.08)\end{array}$ & & MODERATE 1 \\
\hline
\end{tabular}

*Mean difference in serum phosphate level is expressed as intervention group minus standard care group

Cl: Confidence interval; D: Mean difference; Z: Z-score

\section{GRADE Working Group grades of evidence}

High quality: We are very confident that the true effect lies close to that of the estimate of the effect

Moderate quality: We are moderately confident in the effect estimate: The true effect is likely to be close to the estimate of the effect, but there is a possibility that it is substantially different

Low quality: Our confidence in the effect estimate is limited: The true effect may be substantially different from the estimate of the effect

Very low quality: We have very little confidence in the effect estimate: The true effect is likely to be substantially different from the estimate of effect

${ }^{1}$ No explanation was given on blinding of data collectors and allocation concealment in 4 studies 


\section{Background}

The control of serum phosphate levels in people receiving hemodialysis (HD) continues to be a challenge for practitioners and has clinical significance for patient outcomes. If left untreated, hyperphosphatemia significantly increases the risk of morbidity and mortality in people with end stage kidney disease (ESKD). ${ }^{1}$ Hyperphosphatemia occurs in people with ESKD as a result of the kidneys' reduced ability to excrete an ingested phosphate load. ${ }^{2,3}$ Hyperphosphatemia significantly lowers the serum calcium concentration, stimulating the release of parathyroid hormone (PTH), causing secondary hyperparathyroidism. ${ }^{4}$ In ESKD, hyperphosphatemia also slows the activation of vitamin $D$ impaired absorption of calcium from the gastrointestinal tract, resulting in reduced mobilization of calcium and phosphate from the bones, and causing metabolic bone disease. $^{3-5}$ The reduced mobilization of both phosphate and calcium enables these two minerals to bind together creating a calcium-phosphate product. At a serum concentration of $>1.78 \mathrm{mmol} / \mathrm{L}$ (5.5 $\mathrm{mg} / \mathrm{dL}$ ) the calcium-phosphate product crystallizes and is deposited within the soft tissue and vasculature.4, 6,7 In ESKD the development of vascular calcification of the arterial media (i.e. calciphylaxis) is a major contributing factor of morbidity and mortality. ${ }^{7-10}$

\section{Methods of phosphate control}

Hyperphosphatemia is a well-recognized risk factor for renal osteodystrophy and cardiovascular mortality in people with ESKD. 2, 8, 11 Collectively this is called chronic kidney disease - mineral bone disorder (CKD-MBD). Treatment of hyperphosphatemia requires a combination of dietary phosphate restrictions, use of oral phosphate binder therapy to reduce intestinal phosphate absorption, vitamin D3 (calcitriol) supplementation and adequate dialysis prescription to promote removal of phosphate. ${ }^{1}$, 12-14 The treatment goal is to maintain serum phosphate levels at a near normal range of $0.7-1.6$ $\mathrm{mmol} / \mathrm{L}(3.5-5.5 \mathrm{mg} / \mathrm{dL}) .{ }^{15}$ Sustained control of serum phosphate is a strong predictor of improved survival in people receiving $\mathrm{HD},{ }^{11}$ yet approximately $40 \%$ of patients receiving $\mathrm{HD}$ have a serum phosphate level of $>1.6 \mathrm{mmol} / \mathrm{L}(5.0 \mathrm{mg} / \mathrm{dL}) .{ }^{15}$

\section{Dietary phosphate restriction}

The implementation of dietary phosphate restrictions is the first step in treating hyperphosphatemia. The average daily adult diet contains approximately $800-1500 \mathrm{mg}$ of phosphate which is absorbed mostly in the duodenum and jejunum. ${ }^{16}$ However, there is a linear relationship between phosphate and protein intake. ${ }^{2}$ In general, foods high in phosphate such as milk and meat are rich in protein. For each gram of protein, there is approximately $13-15 \mathrm{mg}$ of phosphate. ${ }^{16}$ The recommended protein intake for HD patients is $1.0 \mathrm{~g} / \mathrm{kg}-1.2 \mathrm{~g} / \mathrm{kg}$ ideal body weight per day. ${ }^{17,}{ }^{18}$ However, the dietary restrictions necessary to sustain acceptable serum phosphate in HD patients could lead to protein malnutrition. ${ }^{5,8,19}$ Therefore, dietary phosphate restriction is not sufficient to control serum phosphate levels to current national/international guidelines. ${ }^{19}$ 


\section{Phosphate binders}

The mainstay treatment of hyperphosphatemia is the use of oral phosphate binder medication.2, 20 Phosphate binders lower phosphate absorption in the intestines by binding to and sequestering phosphate in the gastrointestinal tract, forming insoluble products that are not readily absorbed. ${ }^{21}$ The Dialysis Outcomes and Practice Patterns Study (DOPPS) involving 23,898 patients on HD at 923 facilities in 12 countries found that $88 \%$ of patients were prescribed phosphate binders and $12 \%$ were not. There was also a $25 \%$ lower mortality rate $(\mathrm{HR}, 0.75 ; 95 \% \mathrm{Cl} 0.68,0.83)$ in patients who were prescribed phosphate binders versus those not prescribed phosphate binder medication. ${ }^{1}$ However, most people receiving phosphate binding medication do not achieve target serum phosphate level. ${ }^{22}$

\section{Hemodialysis}

Conventional four-hour, thrice-weekly HD removes approximately 700-1,000 mg of phosphate per session, resulting in a weekly reduction of 2,100-3,000 mg.6, 21, 23 Effective phosphate removal through $\mathrm{HD}$ is complicated by phosphate's biphasic elimination from the body. Kinetic studies have shown that serum phosphate levels drop rapidly in the first one to two hours of HD treatment and then reach a plateau. ${ }^{6,23}$ Soon after HD, a rebound of serum phosphate occurs to a rise of about $30-40 \%$. This is because of the slow influx of elements to the extracellular space after a standard HD session. ${ }^{6,22,23}$ It has been recognized that increasing dialysis frequency or duration leads to reduced serum phosphate. ${ }^{19}$ Data from the Frequent Haemodialysis Network nocturnal trial showed that daily or extended nocturnal HD led to an average decrease in serum phosphate levels of $0.4 \mathrm{mmol} / \mathrm{L}(95 \% \mathrm{Cl}$ 0.3, $0.7 \mathrm{mmol} / \mathrm{L}$ ) compared with conventional four-hour thrice-weekly HD treatment. ${ }^{24} \mathrm{HD}$ alone cannot correct the phosphate levels that are associated with the usual dietary content of phosphate in ESKD. ${ }^{21,23}$ Therefore, successful treatment of hyperphosphatemia requires adherence to a combination of dietary phosphate restrictions, ${ }^{6}$ use of oral phosphate binder therapy to reduce absorption ${ }^{21}$ and adequate HD to promote removal. ${ }^{25}$

\section{Adherence to phosphate control}

Research on patient adherence to phosphate control dates back to $1960 .{ }^{13}$ However, the use of education and behavioral interventions to improve long term adherence to phosphate control has been subjected to less investigation in people with ESKD. Several research studies indicate education and behavioral interventions could help individuals change their behavior and hence increase adherence to phosphate control. ${ }^{10}, 12,20,26$ A number of studies have used different educational interventions, carried out by either nurses or dieticians. ${ }^{12,13,27,28}$ Also, structured psychological approaches aimed at helping patients to change their beliefs, consequently developing healthy behavior, have been used to produce positive results in improving adherence to diet, medication and HD treatment. ${ }^{28-31}$ These studies illustrate that theoretical and empirically based behavioral approaches are feasible and can result in a positive reduction of serum phosphate level. Therefore, this systematic review is necessary to identify evidence that further develops useful clinical behavioral 
and educational strategies that can sustain long term adherence with phosphate control.

A literature search of the Cochrane Library, the Joanna Briggs Institute (JBI) Database of Systematic Reviews and Implementation Reports, Prospero international prospective register of systematic reviews and the Campbell Collaboration systematic reviews was conducted and three systematic reviews of relevance to this topic were found. ${ }^{32-34}$ The first systematic review included seven RCTs examining education and counselling interventions to improve dietary phosphate adherence in both HD and pre-dialysis patients. ${ }^{32}$ The second review included 18 studies on educational methods to achieve optimal dietary management of hyperphosphatemia in HD patients. ${ }^{33}$ The third review include seven RCTs that focused on the benefits and harm of dietary interventions for preventing and treating chronic kidney disease-mineral and bone disorder (CKD-MBD). ${ }^{34}$ Although these systematic reviews exist, two of the reviews included only interventions targeting dietary control, while the third review was specific to CKD-MBD (a consequence of long term hyperphosphatemia). This review was needed for several reasons. First, for successful treatment of hyperphosphatemia, patients are required to adhere with a combination of dietary phosphate restrictions, phosphate binder medications and HD prescription. ${ }^{35}$ Second, none of the three methods can control phosphate effectively (particularly long term) in isolation. Third, this review had broader inclusion criteria (study design, adherence to dietary phosphate intake, phosphate binder medication and HD treatment).

\section{Review objective}

Following our published systematic review protocol, ${ }^{36}$ we sought to examine the effectiveness of education or behavioral interventions on adherence to phosphate control in adults with ESKD receiving $\mathrm{HD}$.

\section{Inclusion criteria}

\section{Types of participants}

This review considered studies that included adults over the age of 18 years, with ESKD undergoing HD. Participants were attending dialysis facilities regardless of frequency and duration of treatment sessions per week. Studies with participants receiving hemodiafiltration were excluded.

\section{Types of interventions}

We included studies evaluating education and/or behavioral interventions designed to improve adherence to phosphate control methods (dietary restriction, phosphate binders, HD) as compared to 'routine care (standard care with no formalized, structured or tailored intervention for adherence to phosphate control methods). ${ }^{26,} 28$ Educational interventions are designed to improve patient knowledge and self-management skills, often drawing on behavioral or psychological strategies to empower patients and change behavior. ${ }^{24}$ These interventions can be delivered in combination by any health professional.

\section{Types of outcome measures}

Studies were included that examined one or more of the following primary outcomes: 
- Serum phosphate levels in millimoles per litre $(\mathrm{mmol} / \mathrm{L})$ as measured by laboratory values

- Patient knowledge related to phosphate control as measured by any type of self-report questionnaire

- Patient adherence to phosphate control strategies as measured by tablet counts, electronic monitoring, patient self-report questionnaire, and/or health care professionals' reports

Secondary outcomes also considered included:

- Patient self-management behavior related to phosphate control, as measured by self-report questionnaire

- Patient perceived self-efficacy for phosphate control, as measured by self-report questionnaire

\section{Types of studies}

We included both experimental and observational study designs including randomized controlled trials, non-randomized controlled trials, quasi-experimental studies, before and after studies, prospective and retrospective cohort studies and case control studies.

\section{Search strategy}

The search strategy aimed to find published. A three-step search strategy was used. An initial limited search of CINAHL and MEDLINE was undertaken followed by analysis of the text words contained in the title and abstract, and of the index terms used to describe the article. A second search using all identified keywords and index terms was undertaken across all included databases. Third, the reference list of all identified reports and articles was searched for additional studies. Only studies published in English were considered for inclusion. To ensure the search was comprehensive and covered all the most relevant recent published studies in this topic, studies from the last 10 years (January 2005 to December 2015) were considered for inclusion.

The databases searched included:

- CINAHL

- MEDLINE

- The Cochrane Library

- EMBASE

- Web of Science

- PsycINFO

- ProQuest Dissertations \& Theses Global

Initial keywords used were:

- Kidney failure, end stage kidney disease, ESKD, end stage renal failure, ESRF, chronic kidney disease, CKD, renal insufficiency, chronic renal failure (CRF) or chronic kidney failure (CKF), kidney replacement therapy, KRT, renal replacement therapy, RRT, or hemodialysis 
renal dialysis.

- Health education/program/programme, behavioral/behavioral therapy, cognitive therapy, Knowledge, self-management, knowledge retention, health literacy, self-efficacy.

- Phosphate control, diet therapy, phosphate controlling medication, phosphate binding medication, hyperphosphatemia , adherence, compliance, non-compliance, non-adherence, non-compliant.

Keywords were combined using Boolean, truncation and wildcards operators for the search. An alert was set in each database with the search terms to track newly published articles. The full search strategy is provided in Appendix I.

\section{Methods of the review}

\section{Critical appraisal}

Study titles and abstracts were first screened against the inclusion criteria. Eligible papers selected for retrieval were then assessed by three independent reviewers (MM, $C D \& A B)$ for risk of bias prior to inclusion using standardized critical appraisal instruments from the Joanna Briggs Institute MetaAnalysis of Statistics Assessment and Review Instrument (JBI-MAStARI). Using the JBI-MAStARI critical appraisal instruments (Appendix II) we considered a score of greater than $50 \%$ sufficient quality for inclusion. For RCTs a YES response was required for questions one and six to assess for selection bias, question seven to assess for performance bias and questions eight to ten to assess for detection bias. It is important to note that it is not possible to blind participants (question two) to an education or behavioral intervention. In regards to question three it is not always possible to blind assessors to treatment allocation. Question four (intention to treat) and question five (blinding of outcome assessor), we considered these to be highly desirable for inclusion but not compulsory. For cohort and before-after studies, a YES response was required for question two to assess for appropriate study population, question four and eight to assess for detection bias, questions five and six to assess for selection bias and question nine to assess for appropriate use of statistical analysis. There was no disagreement between reviewers scoring.

\section{Data extraction}

Data were extracted from papers using the standardized data extraction tool from JBI-MAStARI in Appendix III. The data extracted included specific details about the interventions, populations, study methods and outcomes of significance to the review question and specific objectives. The data extracted are detailed in Appendix IV (characteristics of included studies). Three out of the five outcomes listed in the protocol were measured by the included studies: serum phosphate levels, patient knowledge related to phosphate control and perceived self-efficacy for phosphate control. Adherence to diet was measured in seven studies ${ }^{26,37-42}$ four studies measured medications, ${ }^{31,43-45}$ 
six studies measured both diet and medications ${ }^{12,28,29,46-48}$ and no studies measured adherence to HD. No studies measured self-management behaviors. One reviewer extracted the data and verified with a second reviewer.

\section{Data synthesis}

Mean differences $(95 \% \mathrm{Cl})$ and effect size estimates were calculated for continuous outcomes. To enable comparison among studies, all serum phosphate levels were converted to $\mathrm{mmol} / \mathrm{L}$ (conversion ratio: $1 \mathrm{mg} / \mathrm{dL}=3.095 \mathrm{mmol} / \mathrm{L}$ ). ${ }^{33}$ Standardized mean difference (Cohen's $d$ ) effect size was interpreted as: negligible $(<0.2)$, small $(>0.2)$, medium $(>0.5)$ and large $(>0.8) .49$ Data were pooled for meta-analysis using Revman software (Review Manager, version 5.3.4). Meta-analysis of serum phosphate levels using a random-effects model was performed with eight studies because they were comparable in study design, intervention and outcomes. Where the findings could not be pooled using meta-analysis, results are presented in a narrative form.

\section{Description of studies}

A comprehensive database search generated 1,304 citations of which 276 duplicates were removed. The first author reviewed the title and abstracts of the remaining 1,028 articles; 999 were excluded because they did not meet inclusion criteria. The remaining 28 articles appeared to meet the inclusion criteria and were retrieved for full text review. Reference lists were searched and one additional study was found. These 29 studies were reviewed by three reviewers resulting in 11 studies being excluded. Therefore, 18 articles met the inclusion criteria as presented in Figure 1. Appendix IV provides an overview of the characteristics of included articles and Appendix $\mathrm{V}$ provides a list of excluded studies with reasons for exclusion. 


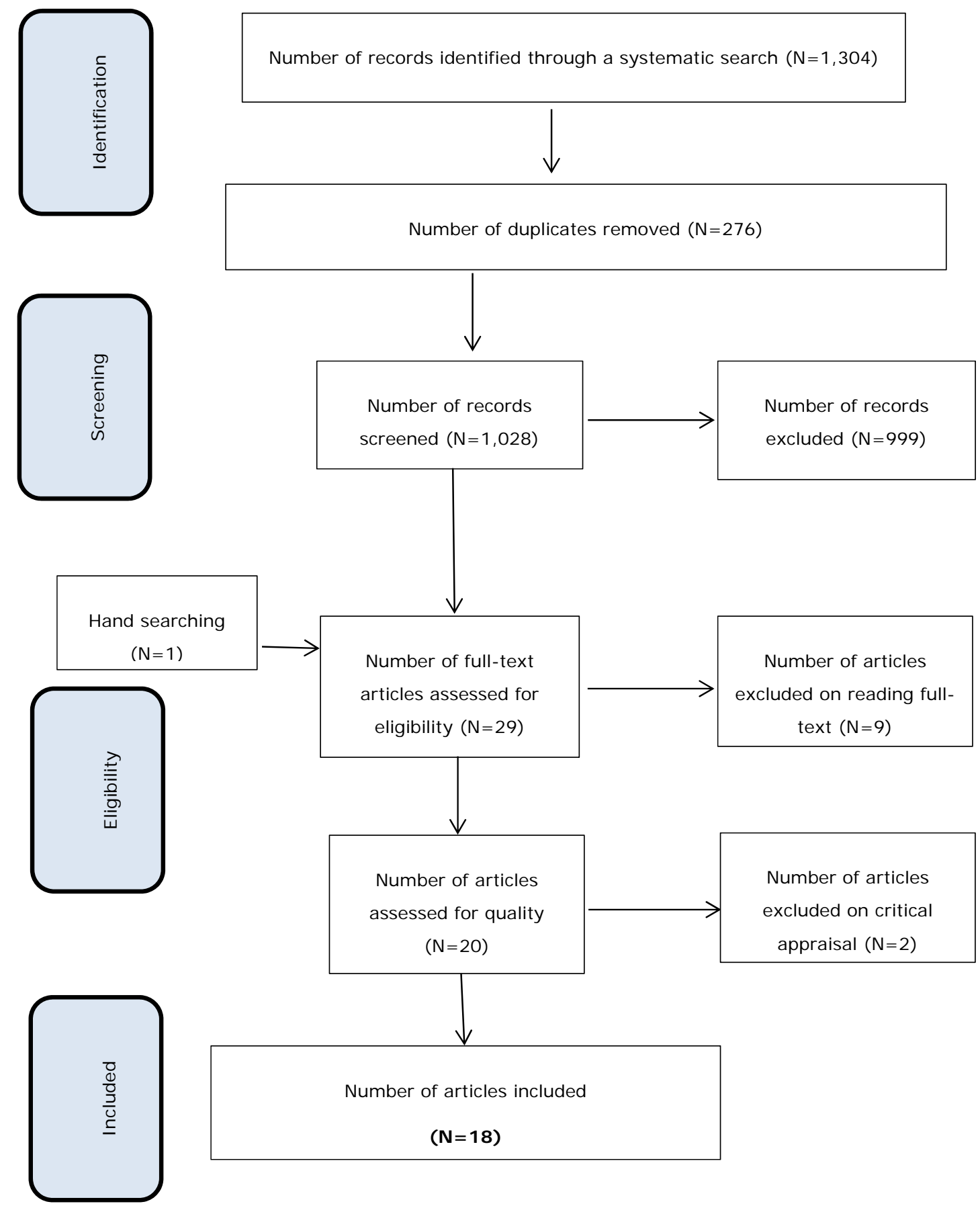

From: Moher D, Liberati A, Tetzlaff J, Altman DG, The PRISMA Group (2009). Preferred Reporting /tems for Systematic Reviews and Meta-Analyses: The PRISMA Statement. PLoS Med 6(6): e1000097. doi:10.1371/journal.pmed1000097

Figure 1: PRISMA flowchart of identified studies for inclusion 
Of the studies included in the review, eight were RCTs, $26,28,38,40,42,43,47,48,50$ nine before-after studies $5,12,29,31,39,41,44-46$ and one cohort study. ${ }^{37} \mathrm{~A}$ total of 2,760 patients were included; 11 studies had more than $50 \%$ males, ${ }^{12}, 28,31,37-40,43,45-47$ four studies had less than $50 \%$ males $26,41,42,44$ and three studies did not provide the sample demographics. ${ }^{5,29,48}$ Sample sizes ranged from 34 to 184 participants per group, although three studies had larger samples: Mayne et $\mathrm{al}^{39}(\mathrm{n}=702)$ and Satoh et $\mathrm{al}^{45}(\mathrm{n}=398)$ did not have control groups, and Van Camp et $\mathrm{al}^{31}$ had unequal group sizes, with 41 in the intervention group and 216 controls. The geographical location of the studies varied, with studies conducted in America, 5, 39, 40, 46 the United Kingdom, 12, 47, 48 Australia, ${ }^{37}, 44$ Belgium, ${ }^{31}$ Sweden, ${ }^{29}$ China, ${ }^{28}$ Japan, ${ }^{45}$ Lebanon, ${ }^{26,}{ }^{42}$ Spain, ${ }^{38}$ and Taiwan. ${ }^{41}$ One study did not mention the country. ${ }^{43}$ Participant inclusion criteria varied between studies: an average monthly serum phosphate

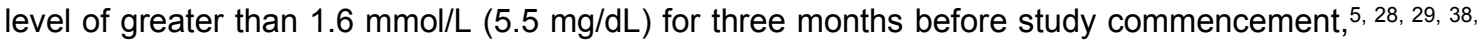
$40,41,48$ currently prescribed phosphate binder medication, $5,28,43,45,46$ or three to six months on HD. ${ }^{28 \text {, }}$ 38,43

\section{Interventions}

Two out of 18 studies used a theoretical framework (self-regulatory) to guide the interventions. ${ }^{26,43}$ Study duration varied between four to 24 weeks with the exception of Campbell et al. ${ }^{37}$ whose study was over two years. Two studies carried out interventions over four weeks, ${ }^{41,} 47$ three studies over eight weeks, ${ }^{26,} 29,40$ one study over 12 weeks $^{44}$, four studies over 16 weeks, ${ }^{43}, 45,46,48$ three studies over 24 weeks, ${ }^{28,38,39}$ one study over 17 weeks ${ }^{31}$ and in two studies it was unclear how long the study took. ${ }^{5,12}$ Educational sessions ranged in numbers from one to 48 during the intervention period and the duration varied from 20 minutes to 60 minutes. In five studies, the intervention took place during the HD session itself. $5,28,31,47,48$ In the other 12 studies it was not stated when the intervention took place.

\section{Educational interventions}

Most of the studies $(n=11)$ used an educational intervention to improve adherence to phosphate control in adults receiving HD. $5,13,38-41,43,46-48$ Different educational techniques aimed at improving both knowledge in phosphate control and achieving acceptable therapeutic biochemistry results were used in various combinations. The educational techniques used varied between studies and included; face to face education, ${ }^{12}, 28,29,37,39-41,43-46$ group education, ${ }^{12}$ watching an educational video, 5,29 listening to audio tapes, ${ }^{12}$ follow-up phone call intervention, ${ }^{40}$ phosphate control educational games, ${ }^{46}$ practical demonstrations ${ }^{43}$ and health promotion literature. ${ }^{12}, 29,39-41,43,45,46$ The education was conducted by several health professionals including nurses, ${ }^{29,41,44}$ dieticians, ${ }^{12,37,39}$ pharmacists, ${ }^{45}$ or interprofessional education (nurse, dietician and nephrologist). ${ }^{29}$

The majority of studies used an individual approach to facilitate learning, $28,37,39-41,44,45$ varying in terms of duration, method of delivery, the facilitator, educational materials used, and follow up period. 
The number of educational sessions ranged from one to 24 and the length from 20 to 30 minutes. Several take home educational materials such as booklets and pamphlets were distributed to patients. In two of the studies the authors did not mention the length of the sessions, follow up period and if any educational material were used. ${ }^{37,44}$ Only one study implemented small group education comprising six to eight patients. Length of educational session was not stated, although the sessions were delivered once a month using booklets and audio cassette with all relevant information translated into local language. ${ }^{12}$

Three studies did not mention which approach was used to deliver the education (individual or group). ${ }^{5,43,46}$ In the first study, Baldwin et al. ${ }^{5}$ used a 45 minute video on diet, medications and HD to control phosphate. In the second study, Karamanidou et al. ${ }^{43}$ delivered a self-regulatory theory-based psycho-educational intervention delivered as a single session, though length of session was not stated. Also, no information is given if the sessions were delivered as individual or group sessions. The third study, Kandiah et al. ${ }^{46}$ implemented a "National Fosphorus League Phootball," game in improving serum phosphate levels as an education strategy. They also put educational literature in the patient waiting room, though it is not mentioned whether the patients took home the educational literature ${ }^{46}$ No information is provided of how, where, when, who, length and how many times the games were played.

\section{Behavioral interventions}

Three studies employed counselling as a behavioral intervention to improve phosphate control. ${ }^{26,38,48}$ In all three studies, dieticians delivered the intervention although one study involved a pharmacist. ${ }^{48}$ Karavetian et al. ${ }^{27}$ conducted individualized, self-management dietary counselling and interactive educational games. The games tested participants' knowledge on dietary phosphate content. Participants were also provided with handouts, booklets of high and low phosphate food alternatives and posters which were placed in the waiting room. ${ }^{26}$ The second study was conducted over 16 weeks with participants receiving individualized consultations every four weeks. No information is given of how, where, when and length of sessions the patients received. ${ }^{48}$ The third study implemented one initial individualized dietary counselling followed by monthly 30 minute sessions to reinforce diet education. ${ }^{38}$

\section{Educational and behavioral interventions}

Three studies used both education and behavioral interventions to improve serum phosphate levels, knowledge and phosphate binder adherence. ${ }^{29}, 31,47$ In all three studies participants received structured educational and counselling sessions. These sessions comprised of group discussions and individual counselling. The interventions were facilitated by specialized nephrology registered nurses, dieticians and nephrologists. There was no consistency between these studies in terms of number of sessions and duration. Sessions lasted on average 20 minutes. In Gardulf et al ${ }^{29}$ the intervention period was eight weeks and sessions were two to three times weekly; on average 60 minute group 
sessions. The sessions consisted of a video presentation on calcium/phosphate balance, provision of brochures on correct dosing of phosphate binders and diet. Patients were asked to write food diaries and these were used to discuss their food intake. In Van camp et al ${ }^{31}$ the intervention period was 17 weeks. Patients received one initial individual educational session and bi-weekly personalized counselling sessions (in weeks 7, 9, 11, 13 and 15). In the last study (Morey et al), ${ }^{47}$ the intervention period was over four weeks, comprising of individualized monthly education but the authors did not report information on length of sessions.

\section{Outcomes}

Serum phosphate level was measured in all studies as an outcome using biochemistry results. Studies collected serum phosphate levels for laboratory analysis at baseline and every month. Baseline serum phosphate level was the value obtained in the month prior to study enrolment and final serum phosphate level was the value obtained at the end of the intervention. Changes in serum phosphate level were calculated using final serum phosphate level minus baseline serum phosphate level in millimoles per litre ( $\mathrm{mmol} / \mathrm{L})$.

Nine studies measured patient knowledge. The studies used pre and post intervention questionnaires for knowledge assessment. Several studies modified previously validated questionnaires to suit their study population, these include modified Cuningham and Llach questionnaire, ${ }^{41}$ Short-Form- $36,{ }^{29}$ Renal Knowledge questionnaire 1998, ${ }^{43}$ Ford et al (2004) questionnaire and School Physical Activity and Nutrition (SPAN) questionnaire (Hoelscher et al. 2003).26, 42 Six studies used self-developed questionnaires which they validated in pilot studies. $26,28,29,40,41,43$ Questionnaires were developed in four studies although none described how validity and reliability were confirmed. 5, 12, 43, 44 The

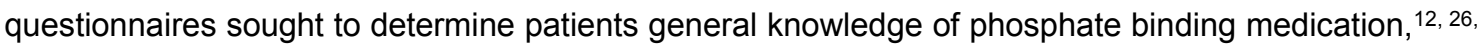
28, 29, 31, 43, 44 diet, ${ }^{12}, 26,28,29,31,40$ adherence to medication, ${ }^{12}, 26,28,44$ phosphate build up and the phosphate binding medications mode of action ${ }^{43}$ and only one study had a questionnaire which included questions on diet, medications and HD to control phosphate. ${ }^{5}$ Two studies explained that post intervention questionnaires were completed at, one month ${ }^{12}$ or immediately after the completion of the four weeks intervention. ${ }^{5}$ Only one study assessed comprehension of the education given. In this study the nurse repeated the education until there was a $100 \%$ pass of the post education evaluation questionnaire. ${ }^{41}$

Adherence to phosphate binding medication and diet restrictions were measured in five studies. Instruments used to measure adherence were the medication event monitoring system (MEMS) ${ }^{31}$ and self-reporting questionnaire. ${ }^{26,43,44,47}$ The authors developed the questionnaires and they did not report how they were validated. No studies collected specific data on two of the outcomes intended to be investigated in this review: self-management and adherence to dialysis.

\section{Methodological quality}

Studies needed to score greater than $50 \%$ based on the primary outcome of serum phosphate level to 
qualify for extraction (Table 2). Overall, RCTs fulfilled a $100 \%$ score in questions one, six to ten, which is assignment to treatment group truly random, groups comparable at entry, groups treated identically other than for the named interventions, outcomes measured in the same and reliable way for all groups and the studies used appropriate statistical analysis. It is unclear if the allocation to treatment groups was concealed from the allocator and if the outcome assessors were blinded to the treatment allocation in some studies therefore, both questions scored $43 \%$. Reporting outcomes for participants who withdraw scored $57 \%$. The overall quality for each study was calculated and ranged from $60 \%$ to $100 \%$. Sample sizes were small in most of the RCTs, ranging from 14 to 41 per group in six studies. $26,28,38,43,47,48$

Quality appraisal for cohort and before-after studies are presented in Table 2. The majority of included studies had appropriate sampling, clear inclusion criteria, adequate follow-up duration, reliable outcome measurement and analysis. All studies assessed were considered to be of sufficient methodological quality for inclusion in the review. The overall quality for each study was calculated and ranged from $67 \%$ to $100 \%$. 
Table 2: Assessment of methodological quality

\begin{tabular}{|c|c|c|c|c|c|c|c|c|c|c|c|}
\hline Study & Q1 & Q2 & Q3 & Q4 & Q5 & Q6 & Q7 & Q8 & Q9 & Q10 & * \\
\hline \multicolumn{12}{|l|}{ RCT } \\
\hline $\begin{array}{l}\text { Karavetian et al.,2015 } \\
42\end{array}$ & $\mathrm{Y}$ & $\mathrm{N} / \mathrm{A}$ & $U$ & $\mathrm{Y}$ & $U$ & $\mathrm{Y}$ & $\mathrm{Y}$ & $\bar{Y}$ & $\mathrm{Y}$ & $\mathrm{Y}$ & 80 \\
\hline $\begin{array}{l}\text { Karavetian et al., } \\
2012^{26}\end{array}$ & $\bar{Y}$ & $\mathrm{~N} / \mathrm{A}$ & $U$ & $\bar{Y}$ & $U$ & $\bar{Y}$ & $\bar{Y}$ & $\bar{Y}$ & $\bar{Y}$ & $\bar{Y}$ & 80 \\
\hline Shi et al., $2013^{28}$ & $\mathrm{Y}$ & $\mathrm{N} / \mathrm{A}$ & $\mathrm{Y}$ & $\bar{Y}$ & $\bar{Y}$ & $\bar{Y}$ & $\bar{Y}$ & $\bar{Y}$ & $\bar{Y}$ & $\bar{Y}$ & 100 \\
\hline Lou et al., $2012^{38}$ & $\mathrm{Y}$ & $\mathrm{N} / \mathrm{A}$ & $\mathrm{Y}$ & $U$ & $U$ & $\mathrm{Y}$ & $\mathrm{Y}$ & $\mathrm{Y}$ & $\mathrm{Y}$ & $\mathrm{Y}$ & 60 \\
\hline Sullivan et al., $2009^{40}$ & $\mathrm{Y}$ & $\mathrm{N} / \mathrm{A}$ & $U$ & $Y$ & $\mathrm{~N}$ & $\bar{Y}$ & $Y$ & $\mathrm{Y}$ & $\mathrm{Y}$ & $\bar{Y}$ & 80 \\
\hline $\begin{array}{l}\text { Karamanidou et al., } \\
2008^{43}\end{array}$ & $\bar{Y}$ & $\mathrm{~N} / \mathrm{A}$ & $U$ & $U$ & $\bar{Y}$ & $\bar{Y}$ & $\bar{Y}$ & $\bar{Y}$ & $\bar{Y}$ & $\bar{Y}$ & 70 \\
\hline Morey et al., $2008^{47}$ & $\mathrm{Y}$ & $\mathrm{N} / \mathrm{A}$ & $U$ & $U$ & $\mathrm{Y}$ & $\mathrm{Y}$ & $\mathrm{Y}$ & $\mathrm{Y}$ & $\mathrm{Y}$ & $\mathrm{Y}$ & 60 \\
\hline Yokum et al., $2008^{48}$ & $\mathrm{Y}$ & $\mathrm{N} / \mathrm{A}$ & $\mathrm{Y}$ & $\mathrm{Y}$ & $U$ & $\mathrm{Y}$ & $\mathrm{Y}$ & $\mathrm{Y}$ & $\mathrm{Y}$ & $\mathrm{Y}$ & 90 \\
\hline$\%$ of criteria met & 100 & 100 & 43 & 57 & 43 & 100 & 100 & 100 & 100 & 100 & \\
\hline \multicolumn{12}{|l|}{ Cohort study } \\
\hline Campbell et al., $2009^{37}$ & $\mathrm{Y}$ & $\mathrm{Y}$ & $\mathrm{U}$ & $\mathrm{Y}$ & $\mathrm{Y}$ & $\mathrm{Y}$ & $\mathrm{Y}$ & $\mathrm{Y}$ & $\mathrm{Y}$ & $\mathrm{Y}$ & 90 \\
\hline
\end{tabular}




\begin{tabular}{|c|c|c|c|c|c|c|c|c|c|c|}
\hline \multicolumn{11}{|l|}{ Before - after study } \\
\hline Baldwin $2013^{5}$ & $\mathrm{Y}$ & $\mathrm{Y}$ & $U$ & $\mathrm{Y}$ & $\mathrm{Y}$ & $\mathrm{Y}$ & $\mathrm{N}$ & $\mathrm{Y}$ & $\mathrm{Y}$ & 75 \\
\hline Sandlin et al., $2013^{44}$ & $\mathrm{Y}$ & $\mathrm{Y}$ & $\mathrm{N}$ & $\mathrm{Y}$ & $\mathrm{Y}$ & $\mathrm{Y}$ & $\mathrm{N}$ & $\mathrm{Y}$ & $\mathrm{Y}$ & 75 \\
\hline Mayne et al., $2012^{39}$ & $\mathrm{~N}$ & $\mathrm{Y}$ & $\mathrm{N}$ & $\mathrm{Y}$ & $\mathrm{Y}$ & $\mathrm{Y}$ & $\mathrm{N}$ & $\mathrm{Y}$ & $\mathrm{Y}$ & 67 \\
\hline Gardulf et al., $2011^{29}$ & $\mathrm{~N}$ & $\mathrm{Y}$ & $\mathrm{Y}$ & $\mathrm{Y}$ & $\mathrm{Y}$ & $\mathrm{Y}$ & $\mathrm{Y}$ & $\mathrm{Y}$ & $\mathrm{Y}$ & 100 \\
\hline Van Camp et al., $2011^{31}$ & $\mathrm{~N}$ & $\mathrm{Y}$ & $U$ & $\mathrm{Y}$ & $\mathrm{Y}$ & $\mathrm{Y}$ & $\mathrm{N}$ & $\mathrm{Y}$ & $\mathrm{Y}$ & 75 \\
\hline Kandiah et al., $2010^{46}$ & $\mathrm{~N}$ & $\mathrm{Y}$ & $\mathrm{N}$ & $\mathrm{Y}$ & $\mathrm{Y}$ & $\mathrm{Y}$ & $\mathrm{N} / \mathrm{A}$ & $\mathrm{Y}$ & $\mathrm{Y}$ & 86 \\
\hline Reddy et al., $2009^{12}$ & $\mathrm{~N}$ & $\mathrm{Y}$ & $\mathrm{N}$ & $\mathrm{Y}$ & $\mathrm{Y}$ & $\mathrm{Y}$ & $\mathrm{Y}$ & $\mathrm{Y}$ & $\mathrm{Y}$ & 86 \\
\hline Satoh et al., $2009^{45}$ & $\mathrm{Y}$ & $\mathrm{Y}$ & $\mathrm{N}$ & $\mathrm{Y}$ & $\mathrm{Y}$ & $\mathrm{Y}$ & $\mathrm{N}$ & $\mathrm{Y}$ & $\mathrm{Y}$ & 75 \\
\hline Sun et al., $2008^{41}$ & $\mathrm{~N}$ & $\mathrm{Y}$ & $\mathrm{N}$ & $\mathrm{Y}$ & $\mathrm{Y}$ & $\mathrm{Y}$ & $\mathrm{N} / \mathrm{A}$ & $\mathrm{Y}$ & $\mathrm{Y}$ & 75 \\
\hline$\%$ of criteria met & 33 & 100 & 44 & 100 & 100 & 100 & 22 & 100 & 100 & \\
\hline
\end{tabular}

Note: $\mathrm{Y}=$ Yes $\quad \mathrm{N}=$ No $\quad \mathrm{U}=$ Unclear $\quad \mathrm{N} / \mathrm{A}=$ Not Applicable due to study design

*Number of applicable criteria met in \% 


\section{Review results}

\section{Serum phosphate levels}

A meta-analysis was conducted to summarize the effectiveness of education or behavioral interventions on serum phosphate levels compared to standard care (See Figure 1 below. Eight RCTs $26,28,38,40,42,43,47,48(n=790)$ were included; 408 in the intervention group and 382 in the standard care group. A random effects model was used because of substantial heterogeneity $\left(x^{2}(7)=26.45, p\right.$ $<0.001, I^{2}=74 \%$ ). Pooled results showed that education or behavioral interventions led to a significant reduction in serum phosphate levels (weighted mean difference $=-0.23 \mathrm{mmol} / \mathrm{L}, 95 \% \mathrm{Cl}=-0.37$, 0.08 ). Sensitivity analyses were performed by excluding studies with substantive methodological differences (i.e. sample size, follow-up period) such as Sullivan et al. ${ }^{40}$ (See appendix VI). Exclusion of Sullivan et al. ${ }^{40}$ strengthened the mean difference $(-0.23 \mathrm{mmol} / \mathrm{L}, 95 \% \mathrm{Cl}=-0.42,-0.05)$, yet heterogeneity remained high $\left(\mathrm{X}^{2}(6)=26.33, p<0.001, \mathrm{I}^{2}=77 \%\right)$.

Studies that were unable to be meaningfully combined for analysis were described in narrative form (Table 3). Eight of the studies reported significant improvement post intervention in phosphate levels achieving the recommended target range of serum phosphate level of 0.7-1.6 mmol/L $(2.17-4.96$ $\mathrm{mg} / \mathrm{dL}){ }^{5}, 12,29,31,37,39,41,45$ The mean serum phosphate improvement varied from $0.3 \mathrm{mmol} / \mathrm{L}$ to 1.0 $\mathrm{mmol} / \mathrm{L}$ (0.93-3.1 $\mathrm{mg} / \mathrm{dL}$ ) and serum phosphate was lower in the education or behavioral intervention group compared to the standard care group. Intervention effect size (ES) varied among studies, one study reported negligible effect, ${ }^{44}$ two studies reported a small effect, ${ }^{41,}{ }^{45}$ three studies reported a medium effect ${ }^{29}, 37,39$ and one study reported a large effect. ${ }^{12}$ Standard clinical measurements provided valid and reliable measures for serum phosphate levels. 
JBI Database of Systematic Reviews \& Implementation Reports

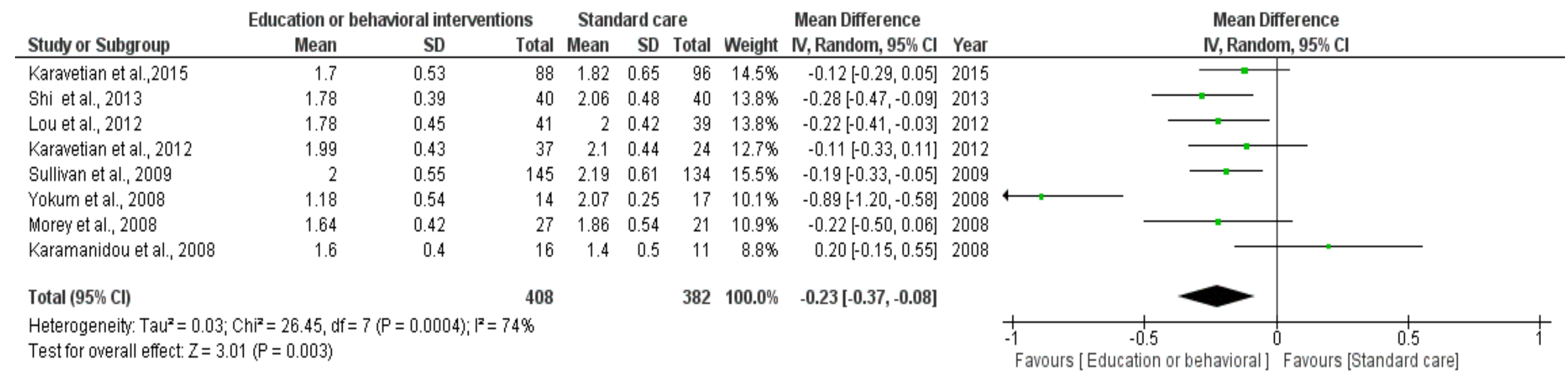

Figure 1: Forest plot of eight experimental studies of educational or behavioral intervention compared with standard care on serum phosphate levels 
Table 3: Effectiveness of education or behavioral interventions on serum phosphate levels in non-RCTs

\begin{tabular}{|c|c|c|c|c|c|c|}
\hline \multirow[b]{2}{*}{$\begin{array}{l}\text { Study } \\
\text { Author }\end{array}$} & \multirow[b]{2}{*}{$\begin{array}{l}\text { Time in } \\
\text { weeks }\end{array}$} & \multicolumn{2}{|c|}{ Intervention group } & \multirow[b]{2}{*}{$\begin{array}{l}\text { Mean } \\
\text { Difference } \\
{[95 \% \mathrm{Cl}]}\end{array}$} & \multirow[b]{2}{*}{$P$-value } & \multirow[b]{2}{*}{$\begin{array}{l}\text { Effect } \\
\text { size }\end{array}$} \\
\hline & & $\begin{array}{l}\text { Baseline } \\
\text { mean } \\
(S D)\end{array}$ & $\begin{array}{l}\text { Final } \\
\text { mean } \\
(S D)\end{array}$ & & & \\
\hline $\begin{array}{l}\text { Baldwin } \\
2013^{5}\end{array}$ & 4 & $\begin{array}{l}2.2 \\
(.33) \\
N=149\end{array}$ & $\begin{array}{l}2.05 \\
(.54) \\
N=148\end{array}$ & $\begin{array}{l}-.15 \\
{[-.25 \text { to }-} \\
.05]\end{array}$ & .0006 & \\
\hline $\begin{array}{l}\text { Sandlin et } \\
\text { al., } 2013^{44}\end{array}$ & 12 & $\begin{array}{l}1.58 \\
(.47) \\
\mathrm{N}=60 \\
\end{array}$ & $\begin{array}{l}1.59 \\
(.44) \\
N=60\end{array}$ & $\begin{array}{l}.01 \\
{[-.15 \text { to } .17]}\end{array}$ & .236 & .02 \\
\hline $\begin{array}{l}\text { Mayne et al., } \\
2012^{39}\end{array}$ & 24 & $\begin{array}{l}1.71 \\
(.06) \\
\mathrm{N}=702\end{array}$ & $\begin{array}{l}1.65 \\
(.1) \\
N=702\end{array}$ & $\begin{array}{l}-.06 \\
{[-.07 \text { to }-} \\
.05]\end{array}$ & $<.01$ & -.73 \\
\hline $\begin{array}{l}\text { Gardulf et } \\
\text { al., } 2011^{29}\end{array}$ & 52 & $\begin{array}{l}2.14 \\
(.56) \\
\mathrm{N}=34\end{array}$ & $\begin{array}{l}1.78 \\
(.50) \\
N=34\end{array}$ & $\begin{array}{l}-.36 \\
{[-.61 \text { to }} \\
.11]\end{array}$ & .01 & -.68 \\
\hline $\begin{array}{l}{ }^{*} \text { Van } \quad \text { Camp } \\
\text { et al., } 2011^{31}\end{array}$ & 17 & $\begin{array}{l}1.58 \\
1.1 \text { to } 2.97 \\
\mathrm{~N}=41\end{array}$ & $\begin{array}{l}1.39 \\
.97 \text { to } 2.1 \\
\mathrm{~N}=216\end{array}$ & & $<.001$ & \\
\hline $\begin{array}{l}\text { Kandiah et } \\
\text { al., } 2010^{46}\end{array}$ & 24 & $\begin{array}{l}1.73 \\
(.55) \\
\mathrm{N}=66\end{array}$ & $\begin{array}{l}1.53 \\
(.57) \\
N=59\end{array}$ & $\begin{array}{l}-.62 \\
{[-1.23 \text { to - }} \\
.01]\end{array}$ & Not given & \\
\hline $\begin{array}{l}\text { Campbell et } \\
\text { al., } 200937\end{array}$ & 104 & $\begin{array}{l}1.8 \\
(.5) \\
\mathrm{N}=33\end{array}$ & $\begin{array}{l}1.5 \\
(.5) \\
N=33\end{array}$ & $\begin{array}{l}-.30 \\
{[-.54 \text { to }-} \\
.06]\end{array}$ & .004 & -.60 \\
\hline $\begin{array}{l}\text { Reddy et al., } \\
2009^{12}\end{array}$ & 20 & $\begin{array}{l}1.55 \\
(.03) \\
\end{array}$ & $\begin{array}{l}1.60 \\
(.04) \\
\end{array}$ & $\begin{array}{l}.05 \\
{[.04 \text { to } .06]}\end{array}$ & .032 & -1.41 \\
\hline
\end{tabular}




\begin{tabular}{|c|c|c|c|c|c|c|}
\hline & & $N=115$ & $N=115$ & & & \\
\hline $\begin{array}{l}{ }^{* * *} \text { Satoh } \quad \text { et } \\
\text { al., } \quad 2009^{45} \\
\text { Levels }<1.29\end{array}$ & 8 & $\begin{array}{l}1.18 \\
(0.08) \\
N=18\end{array}$ & $\begin{array}{l}1.22 \\
(0.23) \\
N=18\end{array}$ & $\begin{array}{l}.04 \\
{[-.07 \text { to } 15]}\end{array}$ & NS & 0.17 \\
\hline $\begin{array}{l}\text { Levels 1.29- } \\
1.58\end{array}$ & & $\begin{array}{l}1.48 \\
(0.08) \\
N=79\end{array}$ & $\begin{array}{l}1.48 \\
(0.2) \\
N=79\end{array}$ & $\begin{array}{l}1.48 \\
(0.2) \\
N=79\end{array}$ & NS & 0.15 \\
\hline $\begin{array}{l}\text { Levels 1.61- } \\
1.91\end{array}$ & & $\begin{array}{l}1.76 \\
(0.09) \\
N=120\end{array}$ & $\begin{array}{l}1.74 \\
(0.2) \\
N=120\end{array}$ & $\begin{array}{l}-.02 \\
{[-.06 \text { to } .02]}\end{array}$ & .01 & 0.16 \\
\hline $\begin{array}{l}\text { Levels } 1.94- \\
2.23\end{array}$ & & $\begin{array}{l}2.07 \\
(0.1) \\
N=68\end{array}$ & $\begin{array}{l}1.99 \\
(0.27) \\
N=68\end{array}$ & $\begin{array}{l}-.08 \\
{[-.15 \text { to }-} \\
.01]\end{array}$ & .005 & 0.20 \\
\hline Levels $\geq 2.26$ & & $\begin{array}{l}2.4 \\
(0.14) \\
N=24\end{array}$ & $\begin{array}{l}2.11 \\
(0.26) \\
N=24\end{array}$ & $\begin{array}{l}-.29 \\
{[-.41 \text { to }-} \\
.17]\end{array}$ & .001 & 0.21 \\
\hline $\begin{array}{l}\text { Sun et al., } \\
2008^{41}\end{array}$ & 12 & $\begin{array}{l}2.38 \\
(.39) \\
N=50\end{array}$ & $\begin{array}{l}2.16 \\
(.64) \\
N=50\end{array}$ & $\begin{array}{l}-.22 \\
{[-.43 \text { to }-} \\
.01]\end{array}$ & .034 & -.43 \\
\hline
\end{tabular}

NS indicates non-significant

* indicates values in mean and range

${ }^{* * *}$ indicates results reported as five sub-groups 


\section{Patient knowledge of phosphate control}

Three RCTs $26,28,40$ and five pre/post intervention 5, 12, 29, 31, 41 studies measured patient knowledge of phosphate control $(n=1,033)$ summarized in Table 4 . All studies reported a significant increase in knowledge scores post intervention. Some studies used pre and post educational serum phosphate levels to evaluate the effectiveness of the patient education given. Patients with improved knowledge were defined as having lower post-education phosphate levels compared to pre - education phosphate levels. 5, 12, 29, 40,41 Combined behavior and knowledge was measured in one study $(n=43)$. The study used a single item ('Food intake associated with phosphate levels, knowledge of the calcium/phosphate balance and knowledge of self-dosing of phosphate binders'). ${ }^{29}$ Participant selfreporting increased significantly in understanding of an appropriate diet to reduce phosphate intake, knowledge about phosphate binders and calcium/phosphate balance after the educational intervention. ${ }^{29}$ Two studies reported a large ES, ${ }^{12}, 28$ two studies reported a small ES ${ }^{26,40}$ and one study reported a moderate ES. ${ }^{42}$ Quality of the included studies was overall good, ranging from $75 \%$ to $100 \%$. However, different questionnaires measuring knowledge were used, and many of these were developed for specific studies, reflecting the lack of appropriate validated questionnaires for the use in this population. Two studies used the same self-report questionnaires to measure knowledge although these were studies by the same author. ${ }^{26,}{ }^{42}$ Therefore, the quality of results for patient knowledge of phosphate control can be rated low grade. 
Table 4: Effectiveness of education or behavioral interventions on patient knowledge of phosphate control

\begin{tabular}{|c|c|c|c|c|c|c|c|}
\hline \multirow[b]{2}{*}{$\begin{array}{l}\text { Study } \\
\text { Author }\end{array}$} & \multirow[b]{2}{*}{$\begin{array}{l}\text { Time in } \\
\text { weeks }\end{array}$} & \multicolumn{2}{|c|}{ Intervention group } & \multicolumn{2}{|c|}{ Standard care group } & \multirow[b]{2}{*}{ P-value } & \multirow[b]{2}{*}{$\begin{array}{l}\text { Effect } \\
\text { size }\end{array}$} \\
\hline & & $\begin{array}{l}\text { Baseline } \\
\text { mean } \\
(S D)\end{array}$ & $\begin{array}{l}\text { Follow up } \\
\text { mean } \\
\text { (SD) }\end{array}$ & $\begin{array}{l}\text { Baseline } \\
\text { mean } \\
(S D)\end{array}$ & $\begin{array}{l}\text { Follow up } \\
\text { mean } \\
\text { (SD) }\end{array}$ & & \\
\hline $\begin{array}{l}\text { Karavetian et } \\
\text { al., } 2015^{42}\end{array}$ & 8 & $\begin{array}{l}40.31 \\
(13.00) \\
\mathrm{N}=88 \\
\end{array}$ & $\begin{array}{l}62.97 \\
(16.89) \\
N=88 \\
\end{array}$ & $\begin{array}{l}38.73 \\
(11.94) \\
N=96\end{array}$ & $\begin{array}{l}51.22 \\
(21.04) \\
N=96 \\
\end{array}$ & $<0.001$ & 0.6 \\
\hline $\begin{array}{l}\text { Baldwin } \\
2013^{5}\end{array}$ & 4 & $\begin{array}{l}5.2 \\
N=149\end{array}$ & $\begin{array}{l}.6 \\
N=148\end{array}$ & & & $<.0001$ & \\
\hline $\begin{array}{l}\text { Karavetian et } \\
\text { al., } 2013^{26}\end{array}$ & 8 & $\begin{array}{l}50 \\
(17) \\
\mathrm{N}=37\end{array}$ & $\begin{array}{l}69 \\
(25) \\
N=37\end{array}$ & $\begin{array}{l}49 \\
(18) \\
N=24\end{array}$ & $\begin{array}{l}61 \\
(29) \\
N=24\end{array}$ & $<.001$ & 24 \\
\hline $\begin{array}{l}\text { Shi et al., } \\
2013^{28}\end{array}$ & 24 & $\begin{array}{l}10.23 \\
(3.91) \\
\mathrm{N}=40\end{array}$ & $\begin{array}{l}23.72 \\
(5.57) \\
N=40\end{array}$ & $\begin{array}{l}9.55 \\
(3.13) \\
N=40\end{array}$ & $\begin{array}{l}16.38 \\
(5.29) \\
N=40\end{array}$ & .05 & 1.16 \\
\hline $\begin{array}{l}\text { Van Camp et } \\
\text { al., } 2011^{31}\end{array}$ & 17 & $\begin{array}{l}53.4 \% \\
(25.0) \\
N=41 \\
\end{array}$ & $\begin{array}{l}75.1 \% \\
(21.7) \\
N=41\end{array}$ & & & $<.0001$ & \\
\hline $\begin{array}{l}\text { Sullivan et } \\
\text { al., } 2009^{40}\end{array}$ & 12 & $\begin{array}{l}67 \\
(16) \\
N=145\end{array}$ & $\begin{array}{l}74 \\
(14) \\
N=145\end{array}$ & $\begin{array}{l}66 \\
(16) \\
N=134\end{array}$ & $\begin{array}{l}70 \\
(16) \\
N=134\end{array}$ & $<.001$ & -.26 \\
\hline $\begin{array}{l}{ }^{* *} \text { Gardulf et } \\
\text { al., } 2011^{29} \\
\text { DIET }\end{array}$ & 52 & $\begin{array}{l}N=22 \\
55 \%\end{array}$ & $\begin{array}{l}N=38 \\
95 \%\end{array}$ & & & $<.001$ & \\
\hline $\begin{array}{l}\text { *Gardulf et } \\
\text { al., 201129 } \\
\text { BINDERS }\end{array}$ & 52 & $\begin{array}{l}N=27 \\
66 \%\end{array}$ & $\begin{array}{l}N=38 \\
93 \%\end{array}$ & & & $<.001$ & \\
\hline $\begin{array}{l}\text { Sullivan et } \\
\text { al., } 2009^{40}\end{array}$ & 12 & $\begin{array}{l}67 \\
(16)\end{array}$ & $\begin{array}{l}74 \\
(14)\end{array}$ & $\begin{array}{l}66 \\
(16)\end{array}$ & $\begin{array}{l}70 \\
(16)\end{array}$ & $<.001$ & -.26 \\
\hline
\end{tabular}




\begin{tabular}{|c|c|c|c|c|c|c|c|}
\hline & & $N=145$ & $N=145$ & $N=134$ & $N=134$ & & \\
\hline $\begin{array}{l}{ }^{* *} \text { Reddy et } \\
\text { al., } 200912 \\
\text { GPK:C }\end{array}$ & 20 & $\begin{array}{l}3.37 \\
(.22) \\
\mathrm{N}=77\end{array}$ & $\begin{array}{l}4.22 \\
(.17) \\
N=77\end{array}$ & & & $<.001$ & .04 \\
\hline $\begin{array}{l}{ }^{* *} \text { Reddy et } \\
\text { al., } 2009^{12} \\
\text { PBK:C }\end{array}$ & 20 & $\begin{array}{l}6.61 \\
(.28) \\
\mathrm{N}=77\end{array}$ & $\begin{array}{l}7.43 \\
(.14) \\
N=77\end{array}$ & & & $<.001$ & 3.70 \\
\hline $\begin{array}{l}{ }^{* *} \text { Reddy et } \\
\text { al., } 2009^{12} \\
\text { GPK:SA }\end{array}$ & 20 & $\begin{array}{l}1.69 \\
(.37) \\
\mathrm{N}=37\end{array}$ & $\begin{array}{l}2.85 \\
(.38) \\
N=37\end{array}$ & & & $<.001$ & 3.10 \\
\hline $\begin{array}{l}{ }^{* *} \text { Reddy et } \\
\text { al., } 2009^{12} \\
\text { PBK:SA }\end{array}$ & 20 & $\begin{array}{l}5.17 \\
(.56) \\
\mathrm{N}=37\end{array}$ & $\begin{array}{l}6.50 \\
(.44) \\
N=37\end{array}$ & & & $<.001$ & 2.64 \\
\hline $\begin{array}{l}\text { Sun et al., } \\
2008^{41}\end{array}$ & 12 & $\begin{array}{l}6.02 \\
(3.61) \\
\mathrm{N}=50\end{array}$ & $\begin{array}{l}\text { All } \\
\text { achieved a } \\
\text { perfect } \\
\text { score }\end{array}$ & & & NS & \\
\hline
\end{tabular}

* indicates data expressed as percentages (\%)

** indicates knowledge reported according to ethnicity

PBK: $\boldsymbol{C}$ indicates knowledge of phosphate binders Caucasian

PBK: SA indicates knowledge of phosphate binders South Asian

GPK: C indicates general knowledge of phosphate diet Caucasian

GPK: SA indicates general knowledge of phosphate diet South Asian

NS indicates non-significant 


\section{Adherence to phosphate control}

Four studies $(n=593)$ reported on adherence to medication ${ }^{31,43,44,47}$ shown in Table 5 and two studies reported on diet restrictions ${ }^{26,38}$ presented in Table 6 . Adherence was measured using the medication event monitoring system (MEMS) ${ }^{31}$ and self-reported questionnaires. ${ }^{26,38,43,44,47}$ Van camp ${ }^{31}$ used MEMS in conjunction with pill count and self-reported questionnaire to measure adherence. They reported an increase in adherence in the intervention group, from a mean of $82.5 \%$ (SD 22.9) to $94.4 \%$ (SD 13.5). Conversely, in the historical control group, adherence declined steadily from a mean of $85.5 \%$ (SD 24.4) in week one to $75.9 \%$ (SD 30.5) in week 14. In studies that used self-reported measures, participants scored how often they failed to take their phosphate binder medication. ${ }^{26,38,43,44,47}$ The studies found no significant group effect change for self-reported patient adherence to phosphate binder medication between the intervention and control groups. Two studies reported a large ES ${ }^{26,43}$ and one study reported a small ES. ${ }^{38}$ Only four out of six studies reported significant increases in adherence with phosphate control. ${ }^{26,31,38,43}$ 
Table 5: Effectiveness of education or behavioral interventions on adherence to phosphate binder medication

\begin{tabular}{|c|c|c|c|c|c|c|c|}
\hline \multirow[b]{2}{*}{$\begin{array}{l}\text { Study } \\
\text { Author }\end{array}$} & \multirow[b]{2}{*}{$\begin{array}{l}\text { Time } \\
\text { in weeks }\end{array}$} & \multicolumn{2}{|c|}{ Intervention group } & \multicolumn{2}{|c|}{ Standard care group } & \multirow[b]{2}{*}{ P-value } & \multirow[b]{2}{*}{$\begin{array}{l}\text { Effect } \\
\text { size }\end{array}$} \\
\hline & & $\begin{array}{l}\text { Baseline } \\
\text { mean } \\
(S D)\end{array}$ & $\begin{array}{l}\text { Follow up } \\
\text { mean } \\
\text { (SD) }\end{array}$ & $\begin{array}{l}\text { Baseline } \\
\text { mean } \\
(S D)\end{array}$ & $\begin{array}{l}\text { Follow up } \\
\text { mean } \\
(S D)\end{array}$ & & \\
\hline $\begin{array}{l}{ }^{1} \text { Sandlin et } \\
\text { al., } 2013^{44}\end{array}$ & 12 & $\begin{array}{l}N=26 \\
44 \%\end{array}$ & $\begin{array}{l}N=26 \\
72 \%\end{array}$ & & & NS & \\
\hline $\begin{array}{l}\text { Van Camp et } \\
\text { al., } 2011^{31}\end{array}$ & 14 & $\begin{array}{l}82.5 \% \\
(22.9) \\
\mathrm{N}=41\end{array}$ & $\begin{array}{l}94.4 \% \\
(13.5) \\
N=41\end{array}$ & $\begin{array}{l}85.5 \% \\
(24.4) \\
N=216 \\
\end{array}$ & $\begin{array}{l}75.9 \% \\
(30.5) \\
N=216 \\
\end{array}$ & .001 & \\
\hline $\begin{array}{l}\text { Karamanidou } \\
\text { et al., } 2008^{43}\end{array}$ & 8 & $\begin{array}{l}2.9 \\
(1.3) \\
N=19\end{array}$ & $\begin{array}{l}2.2 \\
(.9) \\
N=16\end{array}$ & $\begin{array}{l}2.5 \\
(1.4) \\
N=20\end{array}$ & $\begin{array}{l}2.8 \\
(.9) \\
N=11\end{array}$ & .01 & -1.0 \\
\hline $\begin{array}{l}\text { Morey et al., } \\
2008^{47}\end{array}$ & 52 & & & & & NS & \\
\hline
\end{tabular}

1 indicates data expressed as percentages (\%)

NS indicates non-significant

Table 6: Effectiveness of education or behavioral interventions on adherence to diet

\begin{tabular}{|c|c|c|c|c|c|c|c|}
\hline $\begin{array}{l}\text { Karavetian et } \\
\text { al., } 2013^{26} \\
\text { DIET }\end{array}$ & 8 & $\begin{array}{l}21.4 \\
(4.0) \\
\mathrm{N}=37\end{array}$ & $\begin{array}{l}18.3 \\
(2.0) \\
N=37\end{array}$ & $\begin{array}{l}19.5 \\
(2.6) \\
N=24\end{array}$ & $\begin{array}{l}19.8 \\
(3.0) \\
N=24\end{array}$ & .01 & -1.15 \\
\hline $\begin{array}{l}\text { Lou et al., } \\
2012^{38} \\
{ }^{1} \text { DIET }\end{array}$ & 24 & $\begin{array}{l}2.29 \\
(.48) \\
N=41\end{array}$ & $\begin{array}{l}991 \\
(252) \\
N=41\end{array}$ & $\begin{array}{l}1.033 \\
(283) \\
N=39\end{array}$ & $\begin{array}{l}872 \\
(242) \\
N=39\end{array}$ & $<.017$ & .48 \\
\hline
\end{tabular}

1 indicates results in $\mathrm{mg} / 24 \mathrm{~h}$ 


\section{Self-efficacy and self-management behavior}

Only one study ${ }^{43}$ reported on perceived self-efficacy as an outcome measure $(n=39)$ in Table 7 . This study used a single item to measure medication efficacy belief ('how much do you feel you understand about how using phosphate binding medication incorrectly can increase risk of heart or bone disease?'). Participants indicated on a Likert score between one and seven where a higher score was an indication of a strong self-belief. Medication outcome self-efficacy showed significant improvements in the intervention group $(p<.05)$ immediately after the intervention and remained the same, at one and four months later.

No studies collected specific data on self-management. However, seven studies described how at least one self-management component was included. Participants were given simple, specific instructions on how to improve self-management skills such as use of food diaries, ${ }^{29}$ instructions on how to prepare a low phosphate diet, phosphate/protein ratio menus, portion size, following a renalhealthy diet while eating out and at home, ${ }^{38-41}$ how to match phosphate binders to the phosphate content of a meal and meal size, ${ }^{47,} 48$ reading nutrition facts labels. ${ }^{40}$ Overall, the studies demonstrated that providing individuals with self-management strategies was associated with achieving desired behavioral changes and increased self-efficacy. 
Table 7: Effectiveness of education or behavioral interventions on patient perceived selfefficacy

\begin{tabular}{|c|c|c|c|c|c|c|c|}
\hline \multirow[b]{2}{*}{$\begin{array}{l}\text { Study } \\
\text { Author }\end{array}$} & \multirow[b]{2}{*}{$\begin{array}{l}\text { Time in } \\
\text { weeks }\end{array}$} & \multicolumn{2}{|c|}{ Intervention group } & \multicolumn{2}{|c|}{ Standard care group } & \multirow[b]{2}{*}{ P-value } & \multirow[b]{2}{*}{ Effect size } \\
\hline & & $\begin{array}{l}\text { Baseline } \\
\text { mean } \\
\text { (SD) } \\
\text { Number } \\
\text { (N) }\end{array}$ & $\begin{array}{l}\text { Follow up } \\
\text { mean } \\
\text { (SD) } \\
\text { Number } \\
\text { (N) }\end{array}$ & $\begin{array}{l}\text { Baseline } \\
\text { mean } \\
\text { (SD) } \\
\text { Number } \\
\text { (N) }\end{array}$ & $\begin{array}{l}\text { Follow up } \\
\text { mean } \\
\text { (SD) } \\
\text { Number } \\
\text { (N) }\end{array}$ & & \\
\hline $\begin{array}{l}\text { Karamanidou } \\
\text { et al., } 2008^{43}\end{array}$ & 8 & $\begin{array}{l}4.8 \\
(2.2) \\
\mathrm{N}=19\end{array}$ & $\begin{array}{l}6.3 \\
(.7) \\
N=16\end{array}$ & $\begin{array}{l}5.6 \\
(1.7) \\
N=20\end{array}$ & $\begin{array}{l}5.4 \\
(1.5) \\
N=11\end{array}$ & .05 & 1.23 \\
\hline
\end{tabular}




\section{Discussion}

Successful treatment of hyperphosphatemia requires a combination of dietary phosphate restrictions, phosphate binder medications, and HD prescription. ${ }^{12,13,28}$ This review is the first to examine the evidence for educational and behavioral interventions targeting all three methods of phosphate control. Overall, we found moderate strength evidence to support the effectiveness of educational and behavioral interventions in improving serum phosphate levels, patient knowledge and adherence to phosphate control in adults with ESKD who are receiving HD. Meta-analysis of eight RCTs found significant reductions in serum phosphate levels favoring educational or behavioral interventions. However, this result should be interpreted with caution due to the large heterogeneity among studies. These results justify the need for further research to increase the evidence for practice in this area.

Our findings confirm and extend three similar reviews published in 201132, 201433 and 2015. ${ }^{34}$ The first systematic review included seven RCTs of education and counselling interventions to improve dietary phosphate adherence in both HD and pre-dialysis patients. ${ }^{32}$ The second review included 18 studies on educational methods to achieve optimal dietary management of hyperphosphatemia in HD patients. ${ }^{33}$ The third review included nine RCTs that focused on dietary interventions for preventing CKD-MBD. ${ }^{34}$ Though comprehensive, none of these previous systematic reviews considered all three phosphate control methods and largely considered methods within the realm of one discipline (i.e. dietician or pharmacy); therefore making this review necessary. Adherence to all three methods of phosphate control is the standard treatment of hyperphosphatemia in ESKD and does involve the multidisciplinary renal team. ${ }^{35}$ In our review eleven of the studies were also included in the three previous systematic reviews. ${ }^{12,}$ 26, 28, 37-41, 43, 47, 48 However, because we included all phosphate control methods, we found seven additional studies and only one focused on all three methods of phosphate control. ${ }^{5}$ This review highlights that although educational and behavioral interventions targeting dietary phosphate control methods are effective, there is a paucity of quality evidence that reflects standard treatment.

Not all studies gave a detailed description of interventions such as frequency, duration, educational materials used, where, when and how the education was delivered. Methods used to deliver the intervention are important to evaluate the effectiveness of the interventions so that they can be translated into practice. However, difference between the interventions reflects the varied ways health education can be delivered. There is need for high-quality studies that implement well-structured educational methods. This prevents making assumptions of what kind or the method used in the intervention as it renders the work questionable in testing the effectiveness of the education. It is difficult to determine the long term effect of the intervention as only nine studies followed participants for longer than two months. Long term follow up is needed to show whether interventions lead to sustained phosphate control over time. This is important as patients can receive HD for many years, even decades. Nevertheless the studies in this review have reported the benefits of using education as an intervention to improve serum phosphate level. 
Most of the studies used individual educational sessions. This teaching method creates a close relationship between the patient and education provider (e.g. nurse, dietician or pharmacist), allowing for personalized education to take place. However, this type of teaching is time and resource intensive. ${ }^{51-53}$ Research in social psychology has shown the benefits of education delivery in small groups, with participants influencing each other to change their beliefs and behaviors. ${ }^{51,54}$ Only one study in this review provided education in groups. ${ }^{12}$ However, more work is needed even though the effect was consistent reflecting that both individual and group education reduces serum phosphate levels, without either one being superior over the other.

When teaching complex chronic disease management and skills, learning does not always take place. ${ }^{25}$ Evaluating understanding of the education given permits the educator to assess if any changes are needed to be made to promote understanding. Results from these studies seem to indicate that improved understanding on phosphate leads to improved adherence. It is important to include ways of evaluating patient understanding as phosphate control education is complex. ${ }^{55}$ The need for health education on hyperphosphatemia is well documented in the literature. $22,27-29$ Therefore, more studies are needed that evaluate patients understanding of phosphate control education when presented to them, as this might increase understanding and retention resulting in reduced effects of hyperphosphatemia.

None of the studies in this systematic review measured self-management. Individuals with ESKD have a complex chronic disease, often with multiple co morbidities that require long-term selfmanagement. Individuals are faced with daily food choices, taking prescribed medications and attending HD treatment sessions. ${ }^{56}$ Self-management requires clinicians to equip patients with knowledge, skills to care for themselves and to make informed decisions about their health. ${ }^{57}$ If selfmanagement programs are integrated with education of all three methods of phosphate control, there could be improved adherence with phosphate control in individuals with ESKD. A bundled phosphate control educational or behavioral intervention might assist people with ESKD to self-manage, resulting in reduced mortality and complications associated with non-adherence to phosphate control, leading to good clinical outcomes. ${ }^{55}$

Treatment adherence is a continuing problem in the ESKD population ${ }^{13}$ and this review along with the three previous systematic reviews have found little evidence on whether education or behavioral interventions improve adherence with any or all phosphate control methods. Adherence can be affected by educational levels, socio-economic status, multiple co morbidities, the ability to differentiate foods that are a high source of phosphate, ${ }^{58}$ lack of understanding about medications, low health literacy and complexity of the treatment regimen. ${ }^{13,44,59}$ Lack of patient knowledge makes it difficult for many people to comprehend the importance of adhering to the complex ESKD treatment regimens and achieving optimal phosphate levels. ${ }^{60}$ Previous studies have shown that hyperphosphatemia is intensified by poor or non-adherence with diet phosphate restriction, oral phosphate binder medication and HD treatment. ${ }^{28,30,61}$ The negative consequences of non-adherence 
are considerable and can include poor health outcomes and increases morbidity and mortality in ESKD, therefore it is also important for studies to measure adherence.

\section{Limitations}

This systematic review has several limitations. First, the included studies were largely heterogeneous which restricted meta-analysis. Differences in study design, how educational interventions were implemented among studies and outcome measures contributed to the heterogeneity of the studies. In general patient educational programs should be individualized but follow a standardized educational methodology, outcome evaluation and follow up strategies. Heterogeneity in the interventions is a reflection that future studies should design standardized educational programs on adherence to phosphate control in adults receiving HD that can be implemented by other HD units. Other limitations include lack of validated instruments used to evaluate outcomes as most of the studies used self-developed tools to measure various outcomes. Lastly, despite a comprehensive search across electronic databases only studies published in English were included. Research on how to improve adherence to phosphate control in adults receiving HD will need a more detailed and standardized structure of educational methodology and validated instruments to evaluate outcomes.

\section{Conclusion}

This review is the first to include education and behavioral interventions targeting all three phosphate control methods, which reflects practice guidelines internationally. However, most studies in this review intervene on only one facet of phosphate control. Nevertheless our meta-analysis found that educational or behavioral interventions are likely to improve phosphate control among adults with ESKD. Currently there is limited evidence on patient knowledge, adherence and perceived selfefficacy and how these are important for phosphate control. Further studies targeting all three methods of phosphate control are required to increase the level of evidence to inform practice.

\section{Implications for practice}

- Randomized control trials of education/behavioral interventions that target all three methods of phosphate control are required to increase the level of evidence to inform practice. Most studies focused on either dietary phosphate or phosphate binder medication.

- Evidence suggests more intensive education given over a longer period (teaching that is repeated/reinforced) is needed to sustain phosphate control adherence.

- A bundle of patient-centred education and/or behavioral support strategies could improve understanding and adherence with dietary phosphate, phosphate binding medications and dialysis treatment regimen. 


\section{Implications for research}

Adherence with dietary restrictions, medications and dialysis treatment lowers morbidity and mortality, reduces hospitalization, improves quality of life, and will contribute to lower healthcare costs. ${ }^{15}$ Welldesigned and appropriately powered studies that evaluate a bundled intervention for phosphate control in ESKD, with longer follow-up periods, measuring a variety of patient outcomes (e.g. serum phosphate, knowledge, adherence, self-efficacy) are required. Validated instruments are needed to measure self-reported outcomes.

\section{Conflict of interest}

No conflict of interest was foreseen.

\section{Acknowledgements}

The authors thank Librarians at QUT Kelvin grove and University of Queensland Herston for assisting in the retrieval of the articles from the selected databases. 


\section{References}

1. Martin P, Wang P, Robinson A, Poole L, Dragone J, Smyth M, et al. Comparison of dietary phosphate absorption after single doses of lanthanum carbonate and sevelamer carbonate in healthy volunteers: A balance study. Am J Kidney Dis. 2011;57(5):700-6.

2. Bhan I. Phosphate management in chronic kidney disease. Curr Opin Nephrol Hypertens. 2014;23(2):174-9.

3. Blokker M. Hyperphosphatemia and its treatment. CANNT Journal. 2008;18(3):26-7.

4. Román-García P, Carrillo-López N, Cannata-Andía JB. Pathogenesis of bone and mineral related disorders in chronic kidney disease: Key role of hyperphosphatemia. J Ren Care. 2009;35:348.

5. Baldwin DM. Viewing an Educational Video Can Improve Phosphorus Control In Patients On Hemodialysis: A Pilot Study. Nephrol Nurs J. 2013;40(5):437-43.

6. Schucker JJ, Ward KE. Hyperphosphatemia and phosphate binders. Am J Health Syst Pharm. 2005;62(22):2355-61.

7. Shioi A, Nishizawa Y. Vascular calcification in chronic kidney disease: Pathogenesis and clinical implications. J Ren Nutr. 2009;19(1):78-81.

8. Martins MTS, Silva LF, Kraychete A, Reis D, Dias L, Schnitman G, et al. Potentially modifiable factors associated with non-adherence to phosphate binder use in patients on hemodialysis. BMC Nephrol. 2013;14:208-.

9. Navaneethan SD, Palmer SC, Craig JC, Elder GJ, Strippoli GF. Benefits and harms of phosphate binders in CKD: A systematic review of randomized controlled trials. Am J Kidney Dis. 2009;54(4):619-37.

10. Pollock JB, Jaffery JB. Knowledge of phosphorus compared with other nutrients in maintenance dialysis patients. J Ren Nutr. 2007;17(5):323-8.

11. Lopes AA, Tong L, Thumma J, Li Y, Fuller DS, Morgenstern H, et al. Phosphate binder use and mortality among hemodialysis patients in the Dialysis outcomes and practice patterns study (DOPPS): Evaluation of possible confounding by nutritional status. Am J Kidney Dis. 2012;60(1):90101.

12. Reddy V, Symes F, Sethi N, Scally AJ, Scott J, Mumtaz R, et al. Dietitian-led education program to improve phosphate control in a single-center hemodialysis population. J Ren Nutr. 2009;19(4):314-20.

13. San Miguel S, Curtale M, Knagge D, Nhan C, Chow J. Improving patient understanding of phosphate binders: A bony challenge Renal Society of Australasia Journal. 2009;5(3):119-25.

14. Sehgal AR, Sullivan C, Leon JB, Bialostosky K. Public health approach to addressing hyperphosphatemia among dialysis patients. J Ren Nutr. 2008;18(3):256-61.

15. Fouque D, Casal MC, Lindley E, Rogers S, Pancíová J, Kernc J, et al. Dietary trends and management of hyperphosphatemia among patients with chronic kidney disease: An international survey of renal care professionals. J Ren Nutr. 2014;24(2):110-5.

16. González-Parra E, Gracia-Iguacel C, Egido J, Ortiz A. Phosphorus and nutrition in chronic kidney disease. Int J Nephrol. 2012:597605.

17. Naylor H, Jackson H, Walker G, Macafee S, Magee K, Hooper L, et al. British Dietetic Association evidence-based guidelines for the protein requirements of adults undergoing maintenance haemodialysis or peritoneal dialysis. J Hum Nutr Diet. 2013;26(4):315-28.

18. Ash S, Campbell KL, Bogard J, Millichamp A. Nutrition prescription to achieve positive outcomes in chronic kidney disease: A systematic review. Nutrients. 2014;6(1):416-51. 
19. Salusky IB. A new era in phosphate binder therapy: What are the options? Kidney Int Suppl. 2006(105):S10-S5.

20. Cupisti A, Ferretti V, D'Alessandro C, Petrone I, Di Giorgio A, Meola M, et al. Nutritional knowledge in hemodialysis patients and nurses: Focus on phosphorus. J Ren Nutr. 2012;22(6):541-6.

21. Hutchison AJ. Oral phosphate binders. Kidney Int. 2009;75(9):906-14.

22. Ketteler $\mathrm{M}$, Biggar $\mathrm{PH}$. Use of phosphate binders in chronic kidney disease. Curr Opin Nephrol Hypertens. 2013;22(4):413-20.

23. Lorenzo Sellares V, Torres Ramírez A. Management of hyperphosphataemia in dialysis patients: Role of phosphate binders in the elderly. Drugs Aging. 2004;21(3):153-65.

24. Rocco MV, Lockridge RS, Jr., Beck GJ, Eggers PW, Gassman JJ, Greene T, et al. The effects of frequent nocturnal home hemodialysis: The Frequent Hemodialysis Network Nocturnal Trial. Kidney Int. 2011;80(10):1080-91.

25. Mason J, Khunti K, Stone M, Farooqi A, Carr S. Educational interventions in kidney disease care: A systematic review of randomized trials. Am J Kidney Dis. 2008;51(6):933-51.

26. Karavetian M, Ghaddar S. Nutritional education for the management of osteodystrophy (nemo) in patients on haemodialysis: A randomised controlled trial. J Ren Care. 2013;39(1):19-30.

27. Schlatter S, Ferrans CE. Teaching program effects on high phosphorus levels in patients receiving hemodialysis. ANNA J. 1998;25(1):31-6.

28. Shi Y-X, Fan X-Y, Han H-J, Wu Q-X, Di H-J, Hou Y-H, et al. Effectiveness of a nurse-led intensive educational programme on chronic kidney failure patients with hyperphosphataemia: Randomised controlled trial. J Clin Nurs. 2013;22(7-8):1189-97.

29. Gardulf A, Pålsson M, Nicolay U. Education for dialysis patients lowers long-term phosphate levels and maintains health-related quality of life. Clin Nephrol. 2011;75(4):319-27.

30. Sharp J, Wild MR, Gumley AI, Deighan CJ. A cognitive behavioral group approach to enhance adherence to hemodialysis fluid restrictions: A randomized controlled trial. Am J Kidney Dis. 2005;45(6):1046-57.

31. Van Camp YP, Huybrechts SA, Van Rompaey B, Elseviers MM. Nurse-led education and counselling to enhance adherence to phosphate binders. J Clin Nurs. 2012;21(9/10):1304-13.

32. Caldeira D, Amaral T, David C, Sampaio C. Educational strategies to reduce serum phosphorus in hyperphosphatemic patients with chronic kidney disease: Systematic review with metaanalysis. J Ren Nutr. 2011;21(4):285-94.

33. Karavetian M, de Vries N, Rizk R, Elzein H. Dietary educational interventions for management of hyperphosphatemia in hemodialysis patients: A systematic review and meta-analysis. Nutr Rev. 2014;72(7):471-82.

34. Liu Z, Su G, Guo X, Wu Y, Liu X, Zou C, et al. Dietary interventions for mineral and bone disorder in people with chronic kidney disease. Cochrane Database Syst Rev. 2015(9):CD010350.

35. Galassi A, Cupisti A, Santoro A, Cozzolino M. Phosphate balance in ESRD: Diet, dialysis and binders against the low evident masked pool. J Nephrol. 2014:1-15.

36. Milazi M, Bonner A, Douglas $\mathrm{C}$. The effectiveness of education or behavioral interventions on adherence to phosphate control in adults receiving hemodialysis: A systematic review protocol. JBI Database System Rev Implement Rep. 2015;13(3):91-102.

37. Campbell KL, Ash S, Zabel R, McFarlane C, Juffs P, Bauer JD. Implementation of standardized nutrition guidelines by renal dietitians is associated with improved nutrition status. $\mathrm{J}$ Ren Nutr. 2009;19(2):136-44. 
38. Lou LM, Caverni A, Gimeno JA, Moreno R, Pérez J, Alvarez R, et al. Dietary intervention focused on phosphate intake in hemodialysis patients with hyperphosphoremia. Clin Nephrol. 2012;77(6):476-83.

39. Mayne TJ, Benner D, Ricketts K, Burgess M, Wilson S, Poole L, et al. Results of a pilot program to improve phosphorus outcomes in hemodialysis patients. J Ren Nutr. 2012;22(5):472-9.

40. Sullivan C, Sayre SS, Leon JB, Machekano R, Love TE, Porter D, et al. Effect of food additives on hyperphosphatemia among patients with end-stage renal disease: A randomized controlled trial. J Am Med Assoc. 2009;301(6):629-35.

41. Sun C-Y, Chang K-C, Chen S-H, Chang C-T, Wu M-S. Patient education: An efficient adjuvant therapy for hyperphosphatemia in hemodialysis patients. Ren Fail. 2008;30(1):57-62.

42. Karavetian M, de Vries N, Elzein H, Rizk R, Bechwaty F. Effect of behavioral stage-based nutrition education on management of osteodystrophy among hemodialysis patients, Lebanon. Patient Educ Couns. 2015;98(9):1116-22.

43. Karamanidou C, Weinman J, Horne R. Improving haemodialysis patients' understanding of phosphate-binding medication: A pilot study of a psycho-educational intervention designed to change patients' perceptions of the problem and treatment. Br J Health Psychol. 2008;13(Pt 2):205-14.

44. Sandlin K, Bennett PN, Ockerby C, Corradini A-M. The impact of nurse-led education on haemodialysis patients' phosphate binder medication adherence the impact of nurse-led education on haemodialysis patients' phosphate binder medication adherence. J Ren Care. 2013;39(1):12-8.

45. Satoh M, Koizumi A, Izumi S, Kugoh Y, Kiriyama E, Oguma E, et al. Improvement of hyperphosphatemia following patient education. J Pharm Technol. 2009;25(1):3-9.

46. Kandiah J, Resler J, Amend V. Effects of an innovative educational contest to lower serum phosphorous levels and calcium-phosphorous products in hemodialysis patients. Top Clin Nutr. 2010;25(4):345-50.

47. Morey B, Walker R, Davenport A. More dietetic time, better outcome? A randomized prospective study investigating the effect of more dietetic time on phosphate control in end-stage kidney failure haemodialysis patients. Nephron Clinical Practice. 2008;109(3):c173-c80.

48. Yokum D, Glass G, Cheung CF, Cunningham J, Fan S, Madden AM. Evaluation of a phosphate management protocol to achieve optimum serum phosphate levels in hemodialysis patients. J Ren Nutr. 2008;18(6):521-9.

49. Cohen J. Statistical power analysis for the behavioral sciences. Hillsdale, N.J.: L. Erlbaum Associates; 1988.

50. Reese PP, Mgbako O, Mussell A, Potluri V, Yekta Z, Levsky S, et al. A pilot randomized trial of financial incentives or coaching to lower serum phosphorus in dialysis patients. J Ren Nutr. 2015;25(6):510-7.

51. Slyer JT, R. FL. The effectiveness of group visits for patients with heart failure on knowledge, quality of life, self-care, and readmissions: A systematic review. JBI Database System Rev Implement Rep. 2013;11(7):58-81.

52. Håkanson C, Sahlberg-Blom E, Ternestedt B-M, Nyhlin H. Learning about oneself through others: Experiences of a group-based patient education programme about irritable bowel syndrome. Scand J Caring Sci. 2012;26(4):738-46 9p.

53. Whigham L, Joyce $T$, Harper $G$, Irving $P$, Staudacher $H$, Whelan $K$, et al. Clinical effectiveness and economic costs of group versus one-to-one education for short-chain fermentable carbohydrate restriction (low FODMAP diet) in the management of irritable bowel syndrome. J Hum Nutr Diet. 2015;28(6):687-96. 
54. Idier L, Untas A, Koleck M, Chauveau P, Rascle N. Assessment and effects of Therapeutic Patient Education for patients in hemodialysis: A systematic review. Int $\mathrm{J}$ Nurs Stud. 2011;48(12):1570-86.

55. Griva K, Mooppil N, Seet P, Krishnan D, James H, Newman S. The NKF-NUS hemodialysis trial protocol - A randomized controlled trial to determine the effectiveness of a self management intervention for hemodialysis patients. BMC Nephrol. 2011;12(1):4.

56. Ong SW, Jassal SV, Porter E, Logan AG, Miller JA. Using an electronic self-management tool to support patients with chronic kidney disease (CKD): A CKD clinic self-care model. Semin Dial. 2013;26(2):195-202.

57. Richard CJ. Self-care management in adults undergoing hemodialysis. Nephrol Nurs J. 2006;33(4):387-96.

58. Collinson A, McMullan M, Tse WY, Sadler H. Managing serum phosphate in haemodialysis patients: Time for an innovative approach[quest]. Eur J Clin Nutr. 2014;68(3):392-6.

59. Rifkin DE, Laws MB, Rao M, Balakrishnan VS, Sarnak MJ, Wilson IB. Medication adherence behavior and priorities among older adults with CKD: A semistructured interview study. Am J Kidney Dis. 2010;56(3):439-46.

60. Waheed AA, Pedraza F, Lenz O, Isakova T. Phosphate control in end-stage renal disease: Barriers and opportunities. Nephrol Dial Transplant. 2013;28(12):2961-8.

61. Barnett T, Yoong TL, Pinikahana J, Si-Yen T. Fluid compliance among patients having haemodialysis: Can an educational programme make a difference? J Adv Nurs. 2008;61(3):300-6. 


\section{Appendix I: Search strategy}

\section{CINAHL via Ebscohost}

Search on: 07/06/2016

\begin{tabular}{|c|c|}
\hline Search & Query \\
\hline$\# 1$ & $\begin{array}{l}\text { (MH "Kidney Failure, Chronic+") OR (MH "Renal Insufficiency+") OR (MH "Renal } \\
\text { Insufficiency, Chronic+") }\end{array}$ \\
\hline \#2 & $\begin{array}{l}\text { TI ( "kidney failure" OR "end stage kidney disease" OR ESKD OR "end stage } \\
\text { renal failure" OR ESRF OR end stage kidney failure" OR ESKF OR "chronic } \\
\text { kidney disease" OR CKD OR "renal insufficiency" OR "chronic renal failure" OR } \\
\text { CRF OR "chronic kidney failure" OR CKF OR "chronic renal disease" OR CRD } \\
\text { OR "chronic kidney" OR "chronic renal" OR uraemia OR azetoma ) OR AB ( } \\
\text { "kidney failure" OR "end stage kidney disease" OR ESKD OR "end stage renal } \\
\text { failure" OR ESRF OR end stage kidney failure" OR ESKF OR "chronic kidney } \\
\text { disease" OR CKD OR "renal insufficiency" OR "chronic renal failure" OR CRF OR } \\
\text { "chronic kidney failure" OR CKF OR "chronic renal disease" OR CRD OR "chronic } \\
\text { kidney" OR "chronic renal" OR uraemia OR azetoma }\end{array}$ \\
\hline$\# 3$ & $\begin{array}{l}\text { (MH "Renal Replacement Therapy+") OR (MH "Hemodialysis+") OR (MH } \\
\text { "Dialysis Patients") OR (MH "Dialysis Centres") OR (MH "Hemofiltration+") }\end{array}$ \\
\hline$\# 4$ & $\begin{array}{l}\text { TI ( "Kidney replacement therapy" OR KRT OR "renal replacement therapy" OR } \\
\text { RRT OR hemodialysis OR haemodialysis OR "renal dialysis" OR dialysis OR } \\
\text { hemofiltration OR haemofiltration ) OR AB ( "Kidney replacement therapy" OR } \\
\text { KRT OR "renal replacement therapy" OR RRT OR hemodialysis OR } \\
\text { haemodialysis OR "renal dialysis" OR dialysis OR hemofiltration OR } \\
\text { haemofiltration }\end{array}$ \\
\hline \#5 & S1 OR S2 OR S3 OR S4 \\
\hline \#6 & $\begin{array}{l}\text { (MH "Health Education+") OR (MH "Patient Education+") OR (MH "Knowledge+") } \\
\text { OR (MH "Health Knowledge") OR (MH "Information Literacy") }\end{array}$ \\
\hline$\# 7$ & $\begin{array}{l}\text { TX ("Health education" OR "health education progam" OR "patient education" } \\
\text { OR teach* OR train* OR educat* OR knowledge OR "knowledge retention" OR } \\
\text { "health literacy" OR "treatment information" OR "educat* intervention") OR AB ( } \\
\text { "Health education" OR "health education progam*" OR "patient education" OR } \\
\text { teach* OR train* OR educat* OR knowledge OR "knowledge retention" OR } \\
\text { "health literacy" OR "treatment information" OR "educat* intervention") }\end{array}$ \\
\hline$\# 8$ & $\begin{array}{l}\text { (MH "Self Care+") OR (MH "Self-Efficacy") OR (MH "Cognition+") OR (MH } \\
\text { "Nutritional Counseling") OR (MH "Behavioral Changes") }\end{array}$ \\
\hline$\# 9$ & $\begin{array}{l}\text { TI ( Self-management OR "self management OR "self efficacy" OR self-efficacy } \\
\text { OR behavior* OR behavior* OR cognitive OR counselling OR "behavior change" } \\
\text { OR "nutritional counseling" OR "behavior* intervention" OR "behavior* } \\
\text { intervention") OR AB ( Self-management OR "self management OR "self }\end{array}$ \\
\hline
\end{tabular}




\begin{tabular}{|c|c|}
\hline & $\begin{array}{l}\text { efficacy" OR self-efficacy OR behavior* OR behavior* OR cognitive OR } \\
\text { counselling OR "behavior change" OR "nutritional counseling" OR "behavior* } \\
\text { intervention" OR "behavior* intervention" ) }\end{array}$ \\
\hline \#10 & (MH "Medication Compliance") OR (MH "Patient Compliance+") \\
\hline$\# 11$ & $\begin{array}{l}\text { TI ( Adherence OR compliance OR "non compliance OR "non-compliance OR } \\
\text { "non-adherence" OR non adherence OR "non-compliant" OR non compliant OR } \\
\text { complies OR complied OR adhere OR "medication compliance" OR "patient } \\
\text { compliance" ) OR AB ( Adherence OR compliance OR "non compliance OR "non- } \\
\text { compliance OR "non-adherence" OR non adherence OR "non-compliant" OR non } \\
\text { compliant OR complies OR complied OR adhere OR "medication compliance" } \\
\text { OR "patient compliance" ) }\end{array}$ \\
\hline \#12 & S6 OR S7 OR S8 OR S9 OR S10 OR S11 \\
\hline$\# 13$ & $\begin{array}{l}\text { (MH "Diet Therapy+") OR (MH "Renal Diet") OR (MH "Restricted Diet+") OR (MH } \\
\text { "Diet+") OR (MH "Hyperphosphatemia") OR (MH "Phosphorus") OR (MH } \\
\text { "Phosphates") }\end{array}$ \\
\hline \#14 & $\begin{array}{l}\text { TI ("Phosphate control" OR diet OR "diet therapy" OR "renal diet" OR "phosphate } \\
\text { controlling medication" OR "phosphate binding medication" OR } \\
\text { hyperphosphatemia OR hyperphosphataemia OR phosphorus OR phosphate* } \\
\text { OR hyperphosphat*) OR AB ( "Phosphate control" OR diet OR "diet therapy" OR } \\
\text { "renal diet" OR "phosphate controlling medication" OR "phosphate binding } \\
\text { medication" OR hyperphosphatemia OR hyperphosphataemia OR phosphorus } \\
\text { OR phosphate* OR hyperphosphat*) }\end{array}$ \\
\hline \#15 & S13 OR S14 \\
\hline \#16 & S5 AND S12 AND S15 \\
\hline \multicolumn{2}{|c|}{$\begin{array}{l}\text { Limiters - Published Date: 20050101-20151231; English Language; Peer Reviewed; Human; } \\
\text { Age Groups: All Adult } \\
\text { Search modes - Boolean/Phrase }\end{array}$} \\
\hline
\end{tabular}

\section{Medline via Ebscohost}

Search on: 07/06/2016

\begin{tabular}{|l|l|}
\hline Search & Query \\
\hline$\# 1$ & $\begin{array}{l}\text { (MH "Kidney Failure, Chronic+") OR (MH "Renal Insufficiency+") OR (MH "Renal } \\
\text { Insufficiency, Chronic+") OR (MH "Kidney+") }\end{array}$ \\
\hline$\# 2$ & $\begin{array}{l}\text { AB ( "kidney failure" OR "end stage kidney disease" OR ESKD OR "end stage renal } \\
\text { failure" OR ESRF OR end stage kidney failure" OR ESKF OR "chronic kidney disease" } \\
\text { OR CKD OR "renal insufficiency" OR "chronic renal failure" OR CRF OR "chronic kidney }\end{array}$ \\
\hline
\end{tabular}




\begin{tabular}{|c|c|}
\hline & $\begin{array}{l}\text { failure" OR CKF OR "chronic renal disease" OR CRD OR "chronic kidney" OR "chronic } \\
\text { renal" OR uraemia OR azetoma ) OR TI ( "kidney failure" OR "end stage kidney } \\
\text { disease" OR ESKD OR "end stage renal failure" OR ESRF OR end stage kidney failure" } \\
\text { OR ESKF }\end{array}$ \\
\hline$\# 3$ & $\begin{array}{l}\text { (MH "Renal Replacement Therapy+") OR (MH "Renal Dialysis+") OR (MH } \\
\text { "Hemodialysis Units, Hospital") OR (MH "Hemofiltration+") OR (MH "Kidneys, Artificial") }\end{array}$ \\
\hline$\# 4$ & $\begin{array}{l}\text { AB ( "Kidney replacement therapy" OR KRT OR "renal replacement therapy" OR RRT } \\
\text { OR hemodialysis OR haemodialysis OR "renal dialysis" OR dialysis OR hemofiltration } \\
\text { OR haemofiltration ) OR TI ( "Kidney replacement therapy" OR KRT OR "renal } \\
\text { replacement therapy" OR RRT OR hemodialysis OR haemodialysis OR "renal dialysis" } \\
\text { OR dialysis OR hemofiltration OR haemofiltration) }\end{array}$ \\
\hline$\# 5$ & S1 OR S2 OR S3 OR S4 \\
\hline \#6 & $\begin{array}{l}\text { (MH "Health Education+") OR (MH "Education+") OR (MH "Patient Education as } \\
\text { Topic+") OR (MH "Patients+") OR (MH "Patient Medication Knowledge") OR (MH } \\
\text { "Knowledge") OR (MH "Health Literacy+") }\end{array}$ \\
\hline$\# 7$ & $\begin{array}{l}\text { AB ( "Health education" OR "health education progam*" OR "patient education" OR } \\
\text { teach* OR train* OR educat* OR knowledge OR "knowledge retention" OR "health } \\
\text { literacy" OR "treatment information" OR "educat* intervention") OR TI ( "Health } \\
\text { education" OR "health education progam*" OR "patient education" OR teach* OR train* } \\
\text { OR educat* OR knowledge OR "knowledge retention" OR "health literacy" OR } \\
\text { "treatment information" OR "educat* intervention") }\end{array}$ \\
\hline \#8 & (MH "Self Efficacy") OR (MH "Self Care+") OR (MH "Counseling+") \\
\hline$\# 9$ & $\begin{array}{l}\text { AB ( Self-management OR "self management OR "self efficacy" OR self-efficacy OR } \\
\text { behavior* OR behavior" OR cognitive OR counselling OR "behavior change" OR } \\
\text { "nutritional counseling" OR "behavior* intervention" OR "behavior* intervention") OR TI ( } \\
\text { Self-management OR "self management OR "self efficacy" OR self-efficacy OR } \\
\text { behavior* OR behavior* OR cognitive OR counselling OR "behavior change" OR } \\
\text { "nutritional counseling" OR "behavior* intervention" OR "behavior* intervention") }\end{array}$ \\
\hline
\end{tabular}




\begin{tabular}{|c|c|}
\hline$\# 10$ & (MH "Medication Adherence") OR (MH "Compliance") OR (MH "Patient Compliance+") \\
\hline$\# 11$ & $\begin{array}{l}\text { AB ( Adherence OR compliance OR "non compliance OR "non-compliance OR "non- } \\
\text { adherence" OR non adherence OR "non-compliant" OR non compliant OR complies OR } \\
\text { complied OR adhere OR "medication compliance" OR "patient compliance" ) OR TI ( } \\
\text { Adherence OR compliance OR "non compliance OR "non-compliance OR "non- } \\
\text { adherence" OR non adherence OR "non-compliant" OR non compliant OR complies OR } \\
\text { complied OR adhere OR "medication compliance" OR "patient compliance") }\end{array}$ \\
\hline$\# 12$ & S6 OR S7 OR S8 OR S9 OR S10 OR S11 \\
\hline$\# 13$ & $\begin{array}{l}\text { (MH "Diet+") OR (MH "Diet Therapy+") OR (MH "Phosphorus") OR (MH "Phosphorus, } \\
\text { Dietary") OR (MH "Phosphorus Compounds+") OR (MH "Hyperphosphatemia") OR (MH } \\
\text { "Phosphates+") }\end{array}$ \\
\hline \#14 & $\begin{array}{l}\text { AB ( "Phosphate control" OR diet OR "diet therapy" OR "renal diet" OR "phosphate } \\
\text { controlling medication" OR "phosphate binding medication" OR hyperphosphatemia OR } \\
\text { hyperphosphataemia OR phosphorus OR phosphate* OR hyperphosphat* ) OR TI ( } \\
\text { "Phosphate control" OR diet OR "diet therapy" OR "renal diet" OR "phosphate controlling } \\
\text { medication" OR "phosphate binding medication" OR hyperphosphatemia OR } \\
\text { hyperphosphataemia OR phosphorus OR phosphate* OR hyperphosphat" ) }\end{array}$ \\
\hline \#15 & S13 OR S14 \\
\hline$\# 16$ & S5 AND S12 AND S15 \\
\hline \#17 & S5 AND S12 AND S15 \\
\hline \multicolumn{2}{|c|}{$\begin{array}{l}\text { Limiters - Date of Publication: 20050101-20151231; English Language; Human; Age Related: All } \\
\text { Adult: } 19+\text { years } \\
\text { Search modes - Boolean/Phrase }\end{array}$} \\
\hline
\end{tabular}

\section{The Cochrane Library}

Search on: 07/06/2016

\begin{tabular}{|l|l|}
\hline Search & Query \\
\hline$\# 1$ & $\begin{array}{l}\text { kidney failure or end stage kidney disease or ESKD or end stage renal failure or ESRF } \\
\text { or end stage kidney failure or ESKF or chronic kidney disease or CKD or renal } \\
\text { insufficiency or chronic renal failure or CRF or chronic kidney failure or CKF or chronic } \\
\text { renal disease or CRD or chronic kidney or chronic renal or uraemia or azetoma:ti,ab,kw } \\
\text { Online Publication Date from Jan 2005 to Oct 2014 (Word variations have been } \\
\text { searched }\end{array}$ \\
\hline
\end{tabular}




\begin{tabular}{|c|c|}
\hline$\# 2$ & $\begin{array}{l}\text { Kidney replacement therapy or KRT or renal replacement therapy or RRT or } \\
\text { hemodialysis or haemodialysis or renal dialysis or dialysis or hemofiltration or } \\
\text { haemofiltration:ti,ab,kw (Word variations have been searched }\end{array}$ \\
\hline$\# 3$ & \#1 or \#2 \\
\hline$\# 4$ & $\begin{array}{l}\text { Health education or health education progam* or patient education or teach* or train* or } \\
\text { educat }^{*} \text { or knowledge or knowledge retention or health literacy or treatment information } \\
\text { or educat }^{*} \text { intervention:ti,ab,kw (Word variations have been searched) }\end{array}$ \\
\hline \#5 & $\begin{array}{l}\text { Self-management or self management or self efficacy or self-efficacy or behavior* or } \\
\text { behavior* or cognitive or counseling or behavior change or nutritional counselling or } \\
\text { behavior* intervention:ti,ab,kw (Word variations have been searched) }\end{array}$ \\
\hline \#6 & $\begin{array}{l}\text { Adherence or compliance or non compliance or non-compliance or non-adherence or } \\
\text { non adherence or non-compliant or non compliant or complies or complied or adhere or } \\
\text { medication compliance or patient compliance:ti,ab,kw (Word variations have been } \\
\text { searched) }\end{array}$ \\
\hline \#7 & \#4 OR \#5 OR \#6 \\
\hline$\# 8$ & $\begin{array}{l}\text { Phosphate control or diet or diet therapy or renal diet or phosphate controlling } \\
\text { medication or phosphate binding medication or hyperphosphatemia or } \\
\text { hyperphosphataemia or phosphorus or phosphate* or hyperphosphat*:ti,ab,kw (Word } \\
\text { variations have been searched) }\end{array}$ \\
\hline$\# 9$ & \#3 AND \#7 AND \#8 \\
\hline
\end{tabular}

\section{Embase}

Search on: $07 / 06 / 2016$

\begin{tabular}{|l|l|}
\hline Search & Query \\
\hline$\# 1$ & $\begin{array}{l}\text { 'chronic kidney failure'/exp OR 'end stage renal disease'/exp OR 'kidney failure'/exp OR } \\
\text { 'uremia'/exp }\end{array}$ \\
\hline$\# 2$ & $\begin{array}{l}\text { kidney AND failure OR end AND stage AND kidney AND disease OR eskd OR end AND } \\
\text { stage AND renal AND failure OR esrf OR end AND stage AND kidney AND failure OR } \\
\text { eskf OR chronic AND kidney AND disease OR ckd OR renal AND insufficiency OR } \\
\text { chronic AND renal AND failure OR crf OR chronic AND kidney AND failure OR ckf OR } \\
\text { chronic AND renal AND disease OR crd OR chronic AND kidney OR chronic AND renal }\end{array}$ \\
\hline
\end{tabular}




\begin{tabular}{|c|c|}
\hline & OR uraemia OR azetoma \\
\hline \#3 & $\begin{array}{l}\text { 'renal replacement therapy'/exp OR 'hemodialysis'/exp OR 'hemofiltration'/exp OR 'renal } \\
\text { replacement therapy-dependent renal disease'/exp }\end{array}$ \\
\hline$\# 4$ & $\begin{array}{l}\text { kidney AND replacement AND therapy OR krt OR renal AND replacement AND therapy } \\
\text { OR rrt OR hemodialysis OR haemodialysis OR renal AND dialysis OR dialysis OR } \\
\text { hemofiltration OR haemofiltration }\end{array}$ \\
\hline$\# 5$ & \#1 OR \#2 OR \#4 \\
\hline \#6 & $\begin{array}{l}\text { 'patient education'/exp OR 'health education'/exp OR 'health literacy'/exp OR } \\
\text { 'education'/exp OR 'education program'/exp }\end{array}$ \\
\hline \#7 & $\begin{array}{l}\text { health AND education OR health AND education AND progam* }{ }^{*} \text { patient AND } \\
\text { education OR teach* OR train* OR educat* OR knowledge AND retention OR health } \\
\text { AND literacy OR treatment AND information OR educat* AND intervention }\end{array}$ \\
\hline \#8 & $\begin{array}{l}\text { 'self care'/exp OR 'learning'/exp OR 'cognition'/exp OR 'behavior'/exp OR 'behavior } \\
\text { change'/exp OR 'counseling'/exp OR 'nutritional counseling'/exp OR 'nutrition'/exp OR } \\
\text { 'dietary intake'/exp OR 'diet restriction'/exp OR 'potassium restriction'/exp }\end{array}$ \\
\hline$\# 9$ & $\begin{array}{l}\text { 'self management' OR self AND management OR self AND efficacy OR 'self efficacy' } \\
\text { OR behavior* OR behavior* OR cognitive OR counseling OR behavior AND change OR } \\
\text { nutritional AND counselling OR behavior* AND intervention }\end{array}$ \\
\hline$\# 10$ & 'patient compliance'/exp OR 'medication compliance'/exp OR 'dietary compliance'/exp \\
\hline$\# 11$ & $\begin{array}{l}\text { adherence OR compliance OR non AND compliance OR 'non compliance' OR 'non } \\
\text { adherence' OR non AND adherence OR 'non compliant' OR non AND compliant OR } \\
\text { complies OR complied OR adhere OR medication AND compliance OR patient AND } \\
\text { compliance }\end{array}$ \\
\hline$\# 12$ & \#6 OR \#7 OR \#8 OR \#9 OR \#10 OR \#11 \\
\hline
\end{tabular}




\begin{tabular}{|c|c|}
\hline$\# 13$ & 'phosphate'/exp OR 'hyperphosphatemia'/exp OR 'phosphorus'/exp \\
\hline$\# 14$ & $\begin{array}{l}\text { phosphate AND control OR diet AND therapy OR renal AND diet OR phosphate AND } \\
\text { controlling AND medication OR phosphate AND binding AND medication OR } \\
\text { hyperphosphatemia OR hyperphosphataemia OR phosphorus OR phosphate }{ }^{*} \text { OR } \\
\text { hyperphosphat* }\end{array}$ \\
\hline$\# 15$ & $\# 13$ OR \#14 \\
\hline$\# 16$ & \#5 AND \#12 AND \#15 \\
\hline$\# 17$ & $\begin{array}{l}\text { \#16 AND [adult]/lim AND (2005:py OR 2006:py OR 2007:py OR 2008:py OR 2009:py } \\
\text { OR 2010:py OR 2011:py OR 2012:py OR 2013:py OR 2014:py OR 2015:py) }\end{array}$ \\
\hline$\# 18$ & $\begin{array}{l}\text { \#16 AND [adult]/lim AND (2005:py OR 2006:py OR 2007:py OR 2008:py OR 2009:py } \\
\text { OR 2010:py OR 2011:py OR 2012:py OR 2013:py OR 2014:py OR 2015:py) AND } \\
\text { ('cohort analysis'/de OR 'cross-sectional study'/de OR 'human'/de OR 'prospective } \\
\text { study'/de OR 'randomized controlled trial'/de OR 'retrospective study'/de) AND ('bone } \\
\text { disease'/de OR 'chronic kidney disease'/de OR 'chronic kidney failure'/de OR } \\
\text { 'hyperphosphatemia'/de OR 'kidney failure'/de) }\end{array}$ \\
\hline$\# 19$ & $\begin{array}{l}\text { \#16 AND [adult]/lim AND (2005:py OR 2006:py OR 2007:py OR 2008:py OR 2009:py } \\
\text { OR 2010:py OR 2011:py OR 2012:py OR 2013:py OR 2014:py OR 2015:py) AND } \\
\text { ('cohort analysis'/de OR 'cross-sectional study'/de OR 'human'/de OR 'prospective } \\
\text { study'/de OR 'randomized controlled trial'/de OR 'retrospective study'/de) AND ('bone } \\
\text { disease'/de OR 'chronic kidney disease'/de OR 'chronic kidney failure'/de OR } \\
\text { 'hyperphosphatemia'/de OR 'kidney failure'/de) AND ('phosphate'/de OR 'phosphate } \\
\text { binding agent'/de) }\end{array}$ \\
\hline
\end{tabular}

Web of Science

Search on: 07/06/2016

\begin{tabular}{|l|l|}
\hline Search & Query \\
\hline$\# 1$ & $\begin{array}{c}\text { (kidney failure OR end stage kidney disease OR ESKD OR end stage renal failure OR } \\
\text { ESRF OR end stage kidney failure OR ESKF OR chronic kidney disease OR CKD OR }\end{array}$ \\
\hline
\end{tabular}




\begin{tabular}{|c|c|}
\hline & $\begin{array}{l}\text { renal insufficiency OR chronic renal failure OR CRF OR chronic kidney failure OR CKF } \\
\text { OR chronic renal disease OR CRD OR chronic kidney OR chronic renal OR uraemia } \\
\text { OR azetoma) }\end{array}$ \\
\hline \#2 & $\begin{array}{l}\text { (Health education OR health education progam* OR patient education OR teach* OR } \\
\text { train* OR educat* OR knowledge OR knowledge retention OR health literacy OR } \\
\text { treatment information OR educat* intervention) }\end{array}$ \\
\hline$\# 3$ & $\begin{array}{l}\text { (Self-management OR self management OR self efficacy OR self-efficacy OR behavior } \\
\text { OR behavior* OR cognitive OR counseling OR behavior change OR nutritional } \\
\text { counselling OR behavior* intervention) }\end{array}$ \\
\hline$\# 4$ & $\begin{array}{l}\text { (Adherence OR compliance OR non compliance OR non-compliance OR non- } \\
\text { adherence OR non adherence OR non-compliant OR non compliant OR complies OR } \\
\text { complied OR adhere OR medication compliance OR patient compliance) }\end{array}$ \\
\hline$\# 5$ & \#6 OR \#5 OR \#4 \\
\hline \#6 & $\begin{array}{l}\text { (Phosphate control OR diet OR diet therapy OR renal diet OR phosphate controlling } \\
\text { medication OR phosphate binding medication OR hyperphosphatemia OR } \\
\text { hyperphosphataemia OR phosphorus OR phosphate* OR hyperphosphat*) }\end{array}$ \\
\hline$\# 7$ & \#8 AND \#7 AND \#3 \\
\hline
\end{tabular}

\section{PsycINFO via EBSCOHOST}

Search on: 07/06/2016

\begin{tabular}{|l|l|}
\hline Search & Query \\
\hline$\# 1$ & DE "Kidneys" OR DE "Kidney Diseases" \\
\hline$\# 2$ & $\begin{array}{l}\text { TI ( "kidney failure" OR "end stage kidney disease" OR ESKD OR "end stage renal } \\
\text { failure" OR ESRF OR end stage kidney failure" OR ESKF OR "chronic kidney disease" } \\
\text { OR CKD OR "renal insufficiency" OR "chronic renal failure" OR CRF OR "chronic kidney } \\
\text { failure" OR CKF OR "chronic renal disease" OR CRD OR "chronic kidney" OR "chronic } \\
\text { renal" OR uraemia OR azetoma ) OR AB ( "kidney failure" OR "end stage kidney } \\
\text { disease" OR ESKD OR "end stage renal failure" OR ESRF OR end stage kidney failure" } \\
\text { OR ESKF OR "chronic kidney disease" OR CKD OR "renal insufficiency" OR "chronic } \\
\text { renal failure" OR CRF OR "chronic kidney failure" OR CKF OR "chronic renal disease" } \\
\text { OR CRD OR "chronic kidney" OR "chronic renal" OR uraemia OR azetoma ) }\end{array}$ \\
\hline
\end{tabular}




\begin{tabular}{|c|c|}
\hline \#3 & (DE "Hemodialysis") OR (DE "Dialysis" OR DE "Hemodialysis") \\
\hline$\# 4$ & $\begin{array}{l}\text { TI ( "Kidney replacement therapy" OR KRT OR "renal replacement therapy" OR RRT } \\
\text { OR hemodialysis OR haemodialysis OR "renal dialysis" OR dialysis OR hemofiltration } \\
\text { OR haemofiltration ) OR AB ( "Kidney replacement therapy" OR KRT OR "renal } \\
\text { replacement therapy" OR RRT OR hemodialysis OR haemodialysis OR "renal dialysis" } \\
\text { OR dialysis OR hemofiltration OR haemofiltration ) }\end{array}$ \\
\hline \#5 & S1 OR S2 OR S3 OR S4 \\
\hline \#6 & $\begin{array}{l}\text { ((DE "Health Education" OR DE "Drug Education" OR OR DE "Educational Counseling" } \\
\text { OR DE "Client Education" OR DE "Health Literacy") OR (DE "Retention" OR DE "Recall } \\
\text { (Learning)" OR DE "Recognition (Learning)" OR DE "Reconstruction (Learning)")) OR } \\
\text { (DE "Drug Education") }\end{array}$ \\
\hline \#7 & $\begin{array}{l}\text { TI ( "Health education" OR "health education progam" OR "patient education" OR } \\
\text { teach* OR train* OR educat* OR knowledge OR "knowledge retention" OR "health } \\
\text { literacy" OR "treatment information" OR "educat* intervention" ) OR AB ( "Health } \\
\text { education" OR "health education progam*" OR "patient education" OR teach* OR train* } \\
\text { OR educat* OR knowledge OR "knowledge retention" OR "health literacy" OR } \\
\text { "treatment information" OR "educat* intervention") }\end{array}$ \\
\hline \#8 & $\begin{array}{l}\text { ((((DE "Self Management" OR DE "Self Instructional Training") OR (DE "Self Efficacy")) } \\
\text { OR (DE "Behavior Change")) OR (DE "Educational Counseling")) OR (DE "Client } \\
\text { Education") }\end{array}$ \\
\hline \#9 & $\begin{array}{l}\text { TI ( Self-management OR "self management OR "self efficacy" OR self-efficacy OR } \\
\text { behavior* OR behavior* OR cognitive OR counselling OR "behavior change" OR } \\
\text { "nutritional counseling" OR "behavior* intervention" OR "behavior* intervention") OR AB } \\
\text { ( Self-management OR "self management OR "self efficacy" OR self-efficacy OR } \\
\text { behavior* OR behavior* OR cognitive OR counselling OR "behavior change" OR } \\
\text { "nutritional counseling" OR "behavior* intervention" OR "behavior* intervention") }\end{array}$ \\
\hline$\# 10$ & (DE "Treatment Compliance") OR (DE "Compliance" OR DE "Treatment Compliance") \\
\hline \#11 & $\begin{array}{l}\text { TI ( Adherence OR compliance OR "non compliance OR "non-compliance OR "non- } \\
\text { adherence" OR non adherence OR "non-compliant" OR non compliant OR complies OR } \\
\text { complied OR adhere OR "medication compliance" OR "patient compliance" ) OR AB ( } \\
\text { Adherence OR compliance OR "non compliance OR "non-compliance OR "non- } \\
\text { adherence" OR non adherence OR "non-compliant" OR non compliant OR complies OR } \\
\text { complied OR adhere OR "medication compliance" OR "patient compliance") }\end{array}$ \\
\hline \#12 & S6 OR S7 OR S8 OR S9 OR S10 OR S11 \\
\hline$\#$ \#13 & (DE "Phosphorus") OR (DE "Dietary Restraint") \\
\hline$\# 14$ & $\begin{array}{l}\text { TI "Phosphate control" OR diet OR "diet therapy" OR "renal diet" OR "phosphate } \\
\text { controlling medication" OR "phosphate binding medication" OR hyperphosphatemia OR } \\
\text { hyperphosphataemia OR phosphorus OR phosphate* OR hyperphosphat }{ }^{*}\end{array}$ \\
\hline
\end{tabular}




\begin{tabular}{|l|l|}
\hline$\# 15$ & S13 OR S14 \\
\hline$\# 16$ & S5 AND S12 AND S15 \\
\hline$\# 17$ & S5 AND S12 AND S15 \\
\hline \multicolumn{2}{|l|}{$\begin{array}{l}\text { Limiters - Published Date: 20050101-20151231; English } \\
\text { Search modes - Boolean/Phrase }\end{array}$} \\
\hline
\end{tabular}

\section{ProQuest Dissertations \& Theses Global}

Search on: $07 / 06 / 2016$

\begin{tabular}{|c|c|}
\hline Search & uery \\
\hline$\# 1$ & $\begin{array}{l}\text { ((((kidney failure OR end stage kidney disease OR ESKD OR end stage renal failure OR } \\
\text { ESRF OR end stage kidney failure OR ESKF OR chronic kidney disease OR CKD OR } \\
\text { renal insufficiency OR chronic renal failure OR CRF OR chronic kidney failure OR CKF } \\
\text { OR chronic renal disease OR CRD OR chronic kidney OR chronic renal OR uraemia } \\
\text { OR azetoma) OR (Kidney replacement therapy OR KRT OR renal replacement therapy } \\
\text { OR RRT OR hemodialysis OR haemodialysis OR renal dialysis OR dialysis OR } \\
\text { hemofiltration OR haemofiltration)) }\end{array}$ \\
\hline$\# 2$ & $\begin{array}{l}\text { ((( (Health education OR health education progam* OR patient education OR teach* OR } \\
\text { train* OR educat* OR knowledge OR knowledge retention OR health literacy OR } \\
\text { treatment information OR educat }{ }^{*} \text { intervention) OR (Self-management OR self } \\
\text { management OR self efficacy OR self-efficacy OR behavior* OR behavior* OR cognitive } \\
\text { OR counseling OR behavior change OR nutritional counselling OR behavior } \\
\text { intervention) OR (Adherence OR compliance OR non compliance OR non-compliance } \\
\text { OR non-adherence OR non adherence OR non-compliant OR non compliant OR } \\
\text { complies OR complied OR adhere OR medication compliance OR patient compliance)) }\end{array}$ \\
\hline \#3 & $\begin{array}{l}\text { (Phosphate control OR diet OR diet therapy OR renal diet OR phosphate controlling } \\
\text { medication OR phosphate binding medication OR hyperphosphatemia OR } \\
\text { hyperphosphataemia OR phosphorus OR phosphate* OR hyperphosphat }{ }^{*} \text { ) }\end{array}$ \\
\hline \#4 & $\begin{array}{l}\text { ((((kidney failure OR end stage kidney disease OR ESKD OR end stage renal failure } \\
\text { OR ESRF OR end stage kidney failure OR ESKF OR chronic kidney disease OR CKD } \\
\text { OR renal insufficiency OR chronic renal failure OR CRF OR chronic kidney failure OR } \\
\text { CKF OR chronic renal disease OR CRD OR chronic kidney OR chronic renal OR } \\
\text { uraemia OR azetoma) OR (Kidney replacement therapy OR KRT OR renal replacement } \\
\text { therapy OR RRT OR hemodialysis OR haemodialysis OR renal dialysis OR dialysis OR } \\
\text { hemofiltration OR haemofiltration)) AND (((((Health education OR health education } \\
\text { progam* OR patient education OR teach* OR train* OR educat* OR knowledge OR } \\
\text { knowledge retention OR health literacy OR treatment information OR educat* } \\
\text { intervention) OR (Self-management OR self management OR self efficacy OR self- }\end{array}$ \\
\hline
\end{tabular}




\begin{tabular}{|l|l|}
\hline $\begin{array}{l}\text { efficacy OR behavior* OR behavior* OR cognitive OR counseling OR behavior change } \\
\text { OR nutritional counselling OR behavior* intervention) OR (Adherence OR compliance } \\
\text { OR non compliance OR non-compliance OR non-adherence OR non adherence OR } \\
\text { non-compliant OR non compliant OR complies OR complied OR adhere OR medication } \\
\text { compliance OR patient compliance)) AND ((Phosphate control OR diet OR diet therapy } \\
\text { OR renal diet OR phosphate controlling medication OR phosphate binding medication } \\
\text { OR hyperphosphatemia OR hyperphosphataemia OR phosphorus OR phosphate* OR } \\
\text { hyperphosphat*) }\end{array}$ \\
\hline $\begin{array}{l}\text { Limiters - stype.exact("Dissertations \& Theses")) AND at.exact("Dissertation/Thesis")) AND } \\
\text { la.exact("English") AND Published Date (20050101-20151030) }\end{array}$
\end{tabular}




\section{Appendix II: Appraisal instruments}

MAStARI appraisal instrument

\section{JBI Critical Appraisal Checklist for Randomised Control / Pseudo-randomised Trial}

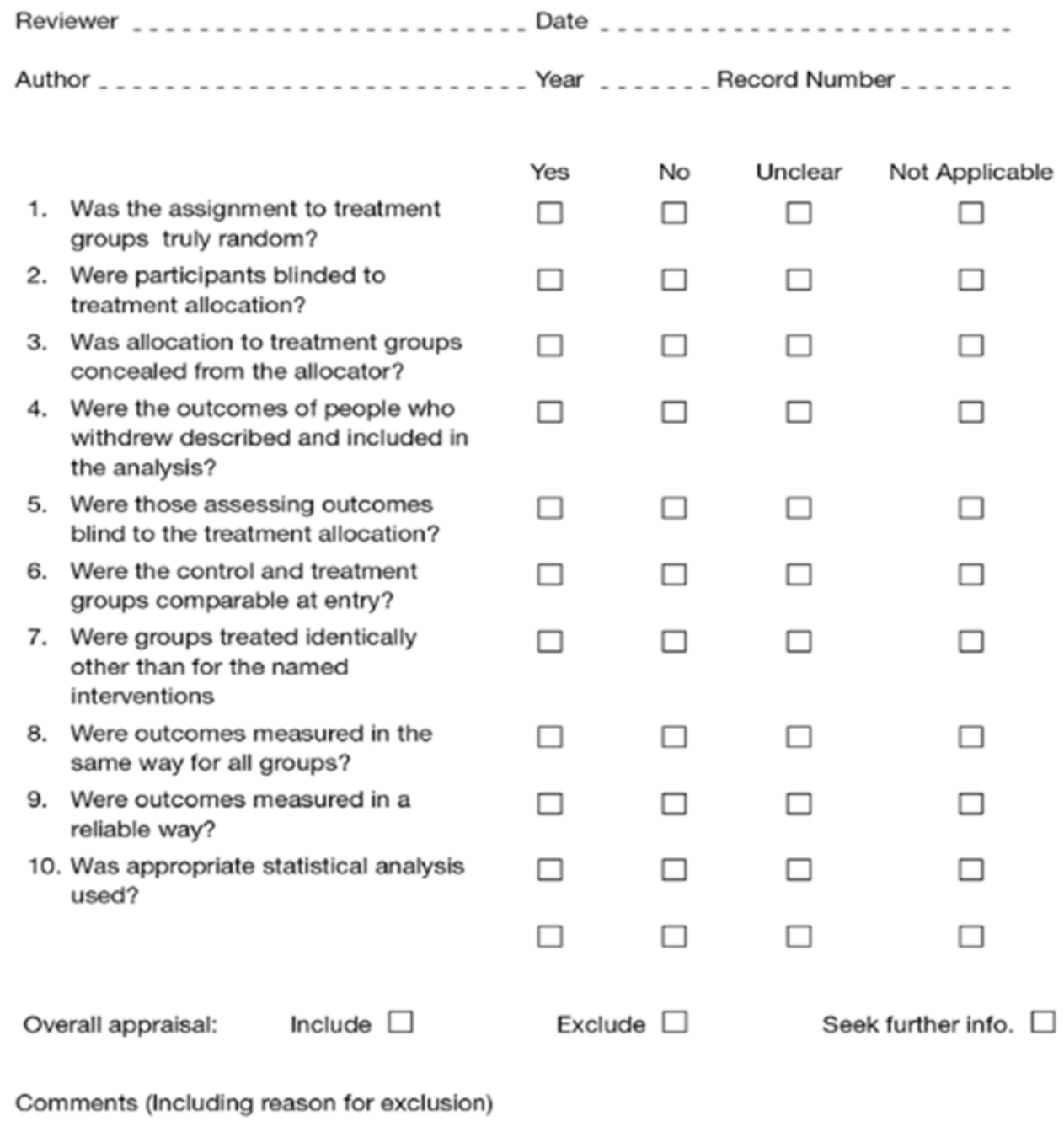




\section{JBI Critical Appraisal Checklist for Descriptive / Case Series}

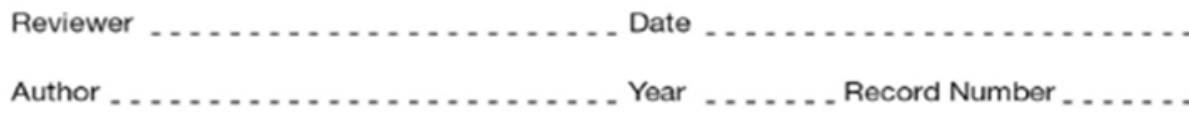

1. Was study based on a random or pseudo-
random sample?

Comments (Including reason for exclusion) 


\section{JBI Critical Appraisal Checklist for Comparable Cohort/ Case Control}

Reviewer .
Author

Overall appraisal: $\quad$ Include $\square \quad$ Exclude $\square \quad$ Seek further info.

Comments (Including reason for exclusion) 


\section{Appendix III: Data extraction instruments}

MAStARI data extraction instrument

\section{JBI Data Extraction Form for Experimental / Observational Studies}

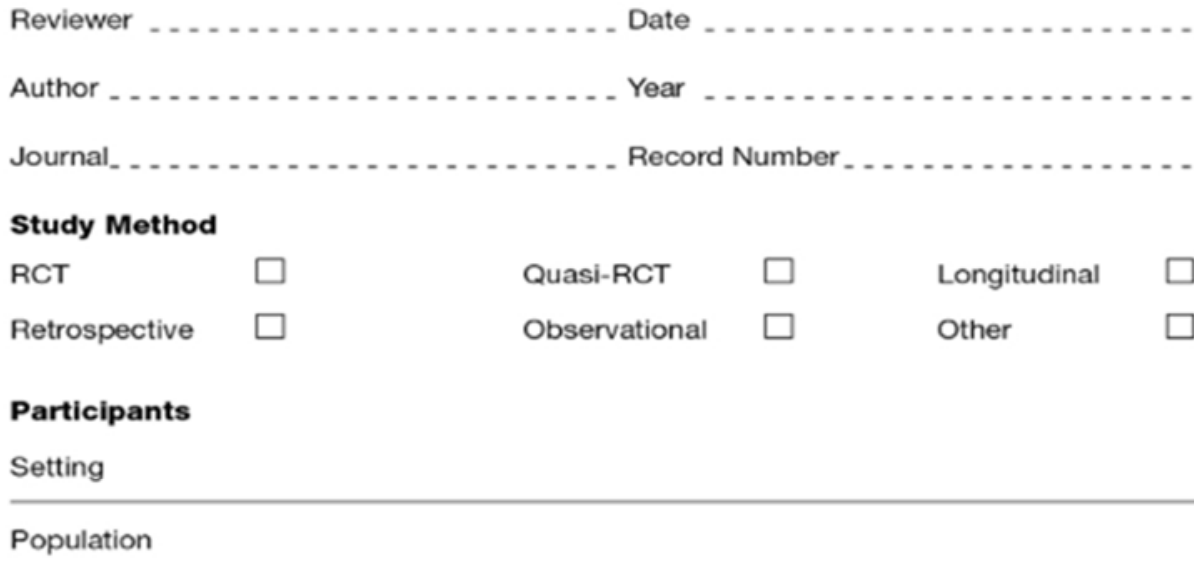

Reviewers Conclusions: 


\section{Study results}

\section{Dichotomous data}

\begin{tabular}{|l|l|l|}
\hline Outcome & $\begin{array}{c}\text { Intervention ( }) \\
\text { number/total number }\end{array}$ & $\begin{array}{c}\text { Intervention ( ) } \\
\text { number/total number }\end{array}$ \\
\hline & & \\
\hline & & \\
\hline & & \\
\hline & & \\
\hline & & \\
\hline
\end{tabular}

Continuous data

\begin{tabular}{|l|l|l|}
\hline Outcome & $\begin{array}{c}\text { Intervention ( ) } \\
\text { number/total number }\end{array}$ & $\begin{array}{c}\text { Intervention ( } \\
\text { number/total number }\end{array}$ \\
\hline & & \\
\hline & & \\
\hline & & \\
\hline & & \\
\hline & & \\
\hline
\end{tabular}




\section{Appendix IV: Characteristics of included studies}

\section{MAStARI}

\begin{tabular}{|c|c|c|c|c|c|}
\hline Authors, year & Study design & $\begin{array}{l}\text { Participant } \\
\text { Sample size = N } \\
\text { Demographics }\end{array}$ & Intervention group & Control group & $\begin{array}{l}\text { Measured outcomes I } \\
\text { Key findings }\end{array}$ \\
\hline $\begin{array}{l}\text { Karavetian et } \\
\text { al., } 2015^{42}\end{array}$ & $\begin{array}{l}\text { Study design: } \\
\text { Randomized control } \\
\text { trial }\end{array}$ & $\begin{array}{l}\text { Inclusion criteria: } \\
\text { Stable on HD for at } \\
\text { least } 3 \text { months, <18 } \\
\text { years of age, Lebanese } \\
\text { origin, full cognitive, } \\
\text { psychiatric and physical } \\
\text { capability for self-care } \\
\text { and communication and } \\
\text { able to provide consent } \\
\text { Intervention: } \mathbf{N}=\mathbf{8 8} \\
\text { Control: } \mathbf{N}=\mathbf{9 6} \\
\mathbf{N}=184 \\
\text { Location : Lebanon }\end{array}$ & $\begin{array}{l}\text { Twice weekly individual nutritional } \\
\text { education for } 15 \text { min during } 6 \\
\text { months, amounting to a total of } \\
12 \text { hours of education per patient } \\
\text {-Other educational tools used were } \\
\text { (1) a renal recipe book tailored to } \\
\text { the Lebanese cuisine, (2) an } \\
\text { illustrated booklet of low phosphate } \\
\text { food options, (3) a poster of low } \\
\text { phosphate foods hung in the unit's } \\
\text { waiting room and (4) an in-centre } \\
\text { patient adherence contest to serum } \\
\text { phosphate } \\
\text { Follow up: None } \\
\text { Educator: Trained dietician by the } \\
\text { study's principal investigator on } \\
\text { renal dietetics }\end{array}$ & $\begin{array}{l}\text { Twice weekly } \\
\text { individual } \\
\text { nutritional } \\
\text { education for } \\
15 \text { min during } \\
6 \text { months, } \\
\text { amounting to a } \\
\text { total of } 12 \\
\text { hours of } \\
\text { education per } \\
\text { patient } \\
\text { - Educational } \\
\text { tools giving at } \\
\text { the end of } \\
\text { intervention } \\
\text { Follow up: } \\
\text { None } \\
\text { Educator: } \\
\text { Trained } \\
\text { hospital } \\
\text { dietitian }\end{array}$ & $\begin{array}{l}\downarrow \text { sig } \mathrm{PO}^{4} \\
\uparrow \text { sig knowledge to acceptable } \\
\text { level at } \mathrm{T} 1 \text {, and was } \\
\text { maintained at } \mathrm{T} 2\end{array}$ \\
\hline Baldwin $2013^{5}$ & $\begin{array}{l}\text { Study design: } \\
\text { Before and after } \\
\text { study }\end{array}$ & $\begin{array}{l}\text { Inclusion criteria: } \\
\text { On HD for at least } 3 \\
\text { months, >18 years, } \\
\text { average monthly } \\
\mathrm{PO}^{4}>\text { than } 1.78 \\
\mathrm{mmol} / \mathrm{L}(5.5 \mathrm{mg} / \mathrm{dL}) \text { for }\end{array}$ & $\begin{array}{l}-45 \text { min educational video } \\
\text { presentation for all patients on diet, } \\
\text { medications and HD to control } \\
\text { phosphate, } 1 \text { to } 2 \text { weeks prior to } \\
\mathrm{PO}^{4} \text { monthly bloods } \\
-72 \text { patients }(52 \%) \text { randomly }\end{array}$ & $\mathbf{N}=\mathbf{0}$ & 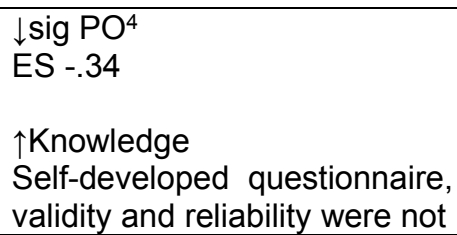 \\
\hline
\end{tabular}




\begin{tabular}{|c|c|c|c|c|c|}
\hline & & $\begin{array}{l}\text { the previous } 3 \text { months, } \\
\text { on phosphate binders } \\
\text { prior to viewing the } \\
\text { video for the first time } \\
\mathbf{N}=150 \\
\text { Demographics not } \\
\text { assessed } \\
\text { Location : United } \\
\text { States }\end{array}$ & $\begin{array}{l}\text { selected for second viewing of the } \\
\text { video before their next blood } \mathrm{PO}^{4} \\
\text { level drawn } \\
\text { Educator : Educational video } \\
\text { Follow up : None }\end{array}$ & & confirmed \\
\hline $\begin{array}{l}\text { Karavetian et } \\
\text { al., } 2013^{26}\end{array}$ & $\begin{array}{l}\text { Study design: } \\
\text { Randomized control } \\
\text { trial }\end{array}$ & $\begin{array}{l}\text { Inclusion criteria: } \\
\text { Stable on HD for at } \\
\text { least } 3 \text { months, <18 } \\
\text { years of age, Lebanese } \\
\text { origin, full cognitive, } \\
\text { psychiatric and physical } \\
\text { capability for self-care } \\
\text { and communication } \\
\text { Intervention: } \mathbf{N}=41 \\
\text { Age in years } \pm \text { SD: } \\
\text { I: } 58 \pm 16 \\
\text { Gender: } \\
\text { Female } 51.5 \% \\
\text { Male } 48.5 \% \\
\text { Control: } \mathbf{N}=40 \\
\text { Age in years } \pm \text { SD: } \\
56 \pm 14 \\
\text { Location : Lebanon }\end{array}$ & $\begin{array}{l}\text { Twice weekly individual nutritional } \\
\text { counselling using interactive games } \\
\text { relevant to weekly educational topic } \\
\text { for } 20 \text { min over } 8 \text { weeks } \\
\text { - Handouts, booklets of high and } \\
\text { low phosphate food alternatives } \\
\text { distributed to participants along with } \\
\text { posters put up in the waiting room } \\
\text { Follow up: None } \\
\text { Educator: Research renal dietician }\end{array}$ & $\begin{array}{l}\text { No nutritional } \\
\text { intervention } \\
\text { Follow up: } \\
\text { None } \\
\text { Educator: } \\
\text { Research } \\
\text { renal dietician }\end{array}$ & $\begin{array}{l}\downarrow \text { sig } \mathrm{PO}^{4} \\
\mathrm{ES}-.74 \\
\uparrow \text { sig Knowledge } \\
\text { ES } .24 \\
\text { Modified previously validated } \\
\text { questionnaires to suit their } \\
\text { study population } \\
\uparrow \text { sig Adherence } \\
\text { ES }-1.15\end{array}$ \\
\hline
\end{tabular}




\begin{tabular}{|c|c|c|c|c|c|}
\hline $\begin{array}{l}\text { Sandlin et al., } \\
2013^{44}\end{array}$ & $\begin{array}{l}\text { Study design: } \\
\text { Before and after } \\
\text { study }\end{array}$ & $\begin{array}{l}\text { Inclusion criteria: } \\
\text { On HD for more than } 1 \\
\text { month and }>18 \text { years of } \\
\text { age } \\
\text { Intervention: } \mathbf{N}=70 \\
\text { Age in years SD: } \\
67.51 \pm 13.35 \\
\text { Gender SD: } \\
\text { Women } 60 \% \\
\text { Male } 40 \% \\
\text { Location } \\
\text { Australia }\end{array}$ & $\begin{array}{l}\text { Intervention group : } \\
\text { Discussions and patient tailored } \\
\text { education over 12-week; author did } \\
\text { not mention length and education } \\
\text { materials used } \\
\text { Follow up: None } \\
\text { Educator: Nurses }\end{array}$ & $\mathbf{N}=\mathbf{0}$ & $\begin{array}{l}\downarrow \mathrm{NS} \mathrm{PO}^{4} \\
\mathrm{ES} .02 \\
\uparrow \text { sig Adherence }\end{array}$ \\
\hline $\begin{array}{l}\text { Shi et al., } \\
2013^{28}\end{array}$ & $\begin{array}{l}\text { Study design: } \\
\text { Randomized control } \\
\text { trail }\end{array}$ & $\begin{array}{l}\text { Inclusion criteria: } \\
\text { Chronic HD } 3 \text { times } \\
\text { weekly for at least } 6 \\
\text { months, aged }>18 \text {, at } \\
\text { latest } 3 \text { month } \mathrm{PO}^{4} \text { and } \\
\text { mean } \mathrm{PO}^{4} \text { both greater } \\
\text { than } 1,78 \mathrm{mmol} / \mathrm{L} \\
\text { (5.5mg/dL) and on } \\
\text { phosphate binders } \\
\text { Intervention: } \mathrm{N}=40 \\
\text { Age in years SD: } \\
54.75 \pm 11.86 \\
\text { Gender: } \\
\text { Woman } 47.5 \% \\
\text { Man } 52.5 \% \\
\text { Control: } \mathrm{N}=40 \\
\text { Age in years SD: } \\
\text { C: } 51.85 \pm 13.51 \\
\text { Gender: } \\
\text { Women } 47.5 \% \\
\text { Man } 52.5 \% \\
\text { Location: China }\end{array}$ & $\begin{array}{l}\text { 2-3 times a week of intensive } \\
\text { individualized education on high- } \\
\text { phosphate foods, phosphate } \\
\text { binders and methods of controlling } \\
\text { phosphate balance over } 24 \text { weeks } \\
\text { for } 20-30 \text { min } \\
\text { - Use of educational booklet; } \\
\text { dialogue, } 1 \text { PowerPoint lecture } \\
\text { (including colourful pictures of high- } \\
\text { phosphate foods, general } \\
\text { phosphate knowledge and } \\
\text { phosphate binders, method to } \\
\text { maintain phosphate balance } \\
\text { Follow up: None } \\
\text { Educator: Trained and } \\
\text { experienced nephrology nurse }\end{array}$ & $\begin{array}{l}\text { Standard } \\
\text { medical and } \\
\text { social care, no } \\
\text { educational } \\
\text { materials } \\
\text { provided } \\
\text { Follow up: } \\
\text { None } \\
\text { Educator: } \\
\text { Trained and } \\
\text { experienced } \\
\text { nephrology } \\
\text { nurse }\end{array}$ & $\begin{array}{l}\downarrow \text { sig } \mathrm{PO}^{4} \\
\mathrm{ES}-.76 \\
\uparrow \text { sig Knowledge } \\
\mathrm{ES} 1.16\end{array}$ \\
\hline
\end{tabular}




\begin{tabular}{|c|c|c|c|c|c|}
\hline $\begin{array}{l}\text { Lou et al., } \\
2012^{38}\end{array}$ & $\begin{array}{l}\text { Study design: } \\
\text { Randomized control } \\
\text { trial }\end{array}$ & $\begin{array}{l}\text { Inclusion criteria: } \\
\text { Age > } 18 \text { years old, } \\
\text { on HD for >6 months, } \\
\text { no acute illness and } \\
\text { Kt/V > 1.2, no feeding } \\
\text { difficulties and normal } \\
\text { appetite, previous 3- } \\
\text { month average PO } \\
>1.78 \text { mmol/L (5.6 } \\
\text { mg/dL) } \\
\text { Intervention: } \mathrm{N}=39 \\
\text { Age in years SD: } \\
63 \pm 16 \\
\text { Gender } \\
\text { Female :46.2\% } \\
\text { Male: } 53.8 \% \\
\text { Control: } \mathrm{N}=41 \\
\text { Age in years SD: } \\
61.3 \pm 15 \\
\text { Gender } \\
\text { Female: } 48.8 \% \\
\text { Male: } 51.2 \% \\
\text { Location: Spain }\end{array}$ & $\begin{array}{l}\text { One initial individualized dietary } \\
\text { counselling followed by monthly } 30 \\
\text { mins reinforcement sessions over } \\
24 \text { weeks } \\
\text { - Instructed on how to prepare low } \\
\text { phosphate and phosphate/protein } \\
\text { ratio menus, food quantities } \\
\text { Follow up: } 6 \text { months } \\
\text { Educator: Registered dietician }\end{array}$ & $\begin{array}{l}\text { Received } \\
\text { usual dietary } \\
\text { recommendati } \\
\text { ons } \\
\text { Follow up: } 6 \\
\text { months } \\
\text { Educator: } \\
\begin{array}{l}\text { Registered } \\
\text { dietician }\end{array}\end{array}$ & $\begin{array}{l}\downarrow \operatorname{sig~} \mathrm{PO}^{4} \\
\mathrm{ES}-.74\end{array}$ \\
\hline $\begin{array}{l}\text { Mayne et al., } \\
2012^{39}\end{array}$ & $\begin{array}{l}\text { Study design: } \\
\text { Before and after } \\
\text { study }\end{array}$ & $\begin{array}{l}\text { Inclusion criteria: } \\
\text { All HD patients } \\
\text { Intervention: } \mathbf{N}=\mathbf{7 0 2} \\
\text { Mean age across all } \\
\text { centres :58 to } 64 \\
\text { Gender: } \\
\text { Male \% across all } \\
\text { centres } \\
43 \% \text { to } 68 \% \\
\text { Location: United States }\end{array}$ & $\begin{array}{l}\text { Single individualized dietary } \\
\text { educational session, study period } \\
24 \text { weeks, length of session not } \\
\text { given } \\
\text { - Patients mailed educational } \\
\text { materials addressing portion size, } \\
\text { following a renal-healthy diet at } \\
\text { home and while eating out } \\
\text { Follow up: } \\
\text { None } \\
\text { Educator: }\end{array}$ & $\mathbf{N}=\mathbf{0}$ & $\begin{array}{l}\downarrow \operatorname{sig} \mathrm{PO}^{4} \\
\mathrm{ES}-.73\end{array}$ \\
\hline
\end{tabular}




\begin{tabular}{|c|c|c|c|c|c|}
\hline & & & Renal dietician & & \\
\hline $\begin{array}{l}\text { Gardulf et al., } \\
2011^{29}\end{array}$ & $\begin{array}{l}\text { Study design: } \\
\text { Before and after } \\
\text { study }\end{array}$ & $\begin{array}{l}\text { Inclusion criteria: } \\
\text { Age <18 years with } \\
\text { CKD Stage } 4 \text { and } 5 \text { with } \\
\text { hyperphosphatemia } \\
\text { despite use of } \\
\text { phosphate binders, } \mathrm{PO}^{4} \\
\text { levels - Stage } 4>1.5 \\
\text { mmol/L ( } 4.6 \mathrm{mg} / \mathrm{dL}) ; \\
\text { CKD Stage } 5>1.8 \\
\text { mmol/L (5.6 mg/dL), } \\
\text { knowledge of the } \\
\text { Swedish language and } \\
\text { no cognitive disorder } \\
\mathrm{N}=43 \\
\text { Age mean (range) } 60.7 \\
\text { (30 - } 82 \text { ) } \\
\text { Gender } \\
\text { Female } 9 \\
\text { Male } 34 \\
\text { Location : Sweden }\end{array}$ & $\begin{array}{l}\text { Education on diet, binders and } \\
\text { calcium/phosphate balance for } 8 \\
\text { weeks; } 2-3 \text { times weekly; on } \\
\text { average } 60 \text { min group sessions } \\
\text { - Video presentation on } \\
\text { calcium/phosphate balance } \\
\text { - Brochures on correct dosing of } \\
\text { phosphate binders and diet } \\
\text { distributed } \\
\text { - Patients asked to write food } \\
\text { diaries and these were used to } \\
\text { discuss their food intake } \\
\text { Educator : Specialized registered } \\
\text { nurses, dietician and nephrologist } \\
\text { Follow up: } 12 \text { months post } \\
\text { intervention }\end{array}$ & $\mathbf{N}=\mathbf{0}$ & $\begin{array}{l}\downarrow \text { sig } \mathrm{PO}^{4}, \\
\text { ES -.68 } \\
\uparrow \text { sig Knowledge } \\
\text { Modified previously validated } \\
\text { questionnaires to suit their } \\
\text { study population }\end{array}$ \\
\hline $\begin{array}{l}\text { Van Camp et } \\
\text { al., 201131 }\end{array}$ & $\begin{array}{l}\text { Study design: } \\
\text { Before and after } \\
\text { study }\end{array}$ & $\begin{array}{l}\text { Inclusion criteria: } \\
\text { Adult patients, on } \\
\text { chronic HD for at least } 3 \\
\text { months, self- } \\
\text { administered medication } \\
\text { care and able to speak } \\
\text { Dutch } \\
\text { Intervention: } \mathbf{N}=41 \\
\text { Age in years [mean } \\
\text { (range)] } 68 \text { (40-83) } \\
\text { Gender \% } \\
\text { Female } 29 \\
\text { Male } 71\end{array}$ & $\begin{array}{l}\text { One initial individual educational } \\
\text { session and bi-weekly personalized } \\
\text { counselling sessions on } \\
\text { hyperphosphatemia and the } \\
\text { importance of phosphate binders in } \\
\text { weeks } 7,9,11,13 \text { and } 15 . \\
\text { Sessions lasted on average } 20 \\
\text { minutes over } 17 \text { weeks } \\
\text { - Patients given pamphlet } \\
\text { Follow up: None } \\
\text { Educator: Study nurse }\end{array}$ & $\begin{array}{l}\text { Not given the } \\
\text { actual } \\
\text { intervention } \\
\text { Follow up: } \\
\text { Not given } \\
\text { Educator: Not } \\
\text { given }\end{array}$ & $\begin{array}{l}\downarrow \text { sig } \mathrm{PO}^{4} \\
\uparrow \text { sig Knowledge } \\
\uparrow \text { sig Adherence }\end{array}$ \\
\hline
\end{tabular}




\begin{tabular}{|c|c|c|c|c|c|}
\hline & & $\begin{array}{l}\text { Historical control } \\
\text { group: } \mathbf{N}=\mathbf{2 1 6} \\
\text { Age in years [mean } \\
\text { (range)] } \\
67(21-90) \\
\text { Gender } \% \\
\text { Female } 43 \\
\text { Male } 57 \\
\text { Location: Belgian }\end{array}$ & & & \\
\hline $\begin{array}{l}\text { Kandiah et al., } \\
2010^{46}\end{array}$ & $\begin{array}{l}\text { Study design: } \\
\text { Before and after } \\
\text { study }\end{array}$ & $\begin{array}{l}\text { Inclusion criteria: } \\
\text { On regular HD, able to } \\
\text { sign an informed } \\
\text { consent } \\
\mathbf{N}=66 \\
\text { Age } \\
18-35-11 \\
36-50-16 \\
51-75-35 \\
>76 \quad-4 \\
\text { Gender } \\
\text { Male - } 33 \\
\text { Female - } 33 \\
\text { Location: Midwestern } \\
\text { dialysis health centre }\end{array}$ & $\begin{array}{l}\text { Study period } 4 \text { month, but not } \\
\text { given length of each educational } \\
\text { session } \\
\text { - Phosphorus football contest and } \\
\text { nutritional educational intervention } \\
\text { Educator: Not mentioned } \\
\text { Follow up: } 6 \text { months post } \\
\text { intervention }\end{array}$ & $\mathbf{N}=0$ & $\begin{array}{l}\downarrow N S \mathrm{PO}^{4} \\
\mathrm{ES}-.36 \\
\text { Implementation of creative } \\
\text { educational strategies may be } \\
\text { effective in improving } \\
\text { biochemical values of HD } \\
\text { patients }\end{array}$ \\
\hline $\begin{array}{l}\text { Campbell et al., } \\
2009^{37}\end{array}$ & $\begin{array}{l}\text { Study design: } \\
\text { Cohort study }\end{array}$ & $\begin{array}{l}\text { Inclusion criteria: } \\
\text { More than } 3 \text { months on } \\
\text { in-centre regular HD (on } \\
\text { average, } 3 \text { times per } \\
\text { week) } \\
N=65 \\
\text { Mean age } \pm \text { SD: } \\
64 \pm 15 \text { years } \\
\text { Location : Australia }\end{array}$ & $\begin{array}{l}2 \text { year study: dietary educational } \\
\text { interviews at the start of study } \\
\text { - follow up afterward, with } \\
\text { reinforcement upon need at least } \\
\text { every } 6 \text { months via individualized } \\
\text { structured nutritional care } \\
\text { - length and educational material } \\
\text { used not mentioned } \\
\text { Follow up: None } \\
\text { Educator : Research renal dietician }\end{array}$ & $\mathbf{N}=\mathbf{0}$ & $\begin{array}{l}\downarrow \text { sig } \mathrm{PO}^{4} \\
\mathrm{ES}-.6 \\
\text { Improved nutritional and } \\
\text { dietary status }\end{array}$ \\
\hline
\end{tabular}




\begin{tabular}{|c|c|c|c|c|c|}
\hline $\begin{array}{l}\text { Reddy et al., } \\
2009^{12}\end{array}$ & $\begin{array}{l}\text { Study design: } \\
\text { Before and after } \\
\text { study }\end{array}$ & $\begin{array}{l}\text { Inclusion criteria: } \\
\text { All HD patients who } \\
\text { consented to participate } \\
\text { in the study } \\
\text { Intervention: } \mathbf{N}=115 \\
\text { Mean age in years } \\
\text { Women } 61.5 \\
\text { Men } 60.3 \\
\text { Gender } \\
\text { Women } 53 \% \\
\text { Men } 62 \% \\
\text { Location: United } \\
\text { Kingdom }\end{array}$ & $\begin{array}{l}\text { Once a month structured group } \\
\text { education of } 6-8 \text { participants } \\
\text {-Education on general knowledge } \\
\text { of phosphate and phosphate } \\
\text { binders, length of each session and } \\
\text { study period not mentioned } \\
\text { - Use of booklets written by a } \\
\text { dietician, along with an audio } \\
\text { cassette on all relevant information, } \\
\text { translated into local language } \\
\text { Follow up: None } \\
\text { Educator: Research dietician }\end{array}$ & $\mathbf{N}=\mathbf{0}$ & $\begin{array}{l}\downarrow \text { sig } \mathrm{PO}^{4} \\
\text { ES } 1.41 \\
\uparrow \text { sig Knowledge } \\
\text { ES } 3.70 \\
\text { Self-developed questionnaire, } \\
\text { validity and reliability were not } \\
\text { confirmed }\end{array}$ \\
\hline $\begin{array}{l}\text { Satoh et al., } \\
200945\end{array}$ & $\begin{array}{l}\text { Study design: } \\
\text { Before and after } \\
\text { study }\end{array}$ & $\begin{array}{l}\text { Inclusion criteria: } \\
\text { All HD patients } \\
\mathbf{N}=398 \\
\text { Demographics not } \\
\text { assessed } \\
\text { Location: Japan }\end{array}$ & $\begin{array}{l}\text { Intervention group } \\
\text { Educational sessions on phosphate } \\
\text { binders and hyperphosphatemia } \\
\text { over } 16 \text { weeks; length of session } \\
\text { was based on the patient's baseline } \\
\text { serum, longer session were } 20-40 \\
\text { mins } \\
\text { - All patients received education in } \\
\text { a verbal and written format once } \\
\text { from the pharmacist } \\
\text { - Patients with PO }{ }^{4} \text { less than } 1.29 \\
\text { mmol/L ( } 4.0 \text { mg/dL) were given only } \\
\text { limited information } \\
\text {-Whilst patients with results greater } \\
\text { than or equal to } 2.26 \text { mmol/L ( } 7.0 \\
\text { mg/dL) received the most } \\
\text { comprehensive explanations } \\
\text { Follow up: } 8 \text { week post } \\
\text { intervention } \\
\text { Educator: Pharmacist }\end{array}$ & $\mathrm{N}=0$ & $\begin{array}{l}\downarrow \text { sig } \mathrm{PO}^{4} \\
\text { ES } \\
\text { Levels }<1.29=0.17 \\
\text { Levels } 1.29-1.58=0.15 \\
\text { Levels } 1.61-1.91=0.16 \\
\text { Levels } 1.94-2.23=0 . .20 \\
\text { Levels } \geq 2.26=0.21\end{array}$ \\
\hline
\end{tabular}




\begin{tabular}{|c|c|c|c|c|c|}
\hline $\begin{array}{l}\text { Sullivan et al., } \\
2009^{40}\end{array}$ & $\begin{array}{l}\text { Study design: } \\
\text { Randomized control } \\
\text { trial }\end{array}$ & $\begin{array}{l}\text { Inclusion criteria: } \\
\text { All } 3 \text { times weekly } \mathrm{HD} \\
\text { patients with, } 3 \text { months } \\
\mathrm{PO}^{4} \text { and mean } \mathrm{PO}^{4} \\
\text { greater than } 1.78 \\
\text { mmol/L (5.5mg/dL) } \\
\text { Intervention: } \mathrm{N}=145 \\
\text { Age in years SD } \\
\text { l: } 54 \pm 13 \\
\text { Gender } \\
\text { Women } 43 \% \\
\text { Man } 57 \% \\
\text { Control: } \mathrm{N}=134 \\
\text { Age in years SD } \\
\text { C: } 52 \pm 13 \\
\text { Gender } \\
\text { Women } 34 \% \\
\text { Man } 66 \% \\
\text { Location: USA }\end{array}$ & $\begin{array}{l}\text { Single individualized educational } \\
\text { session on avoiding foods with } \\
\text { phosphate additives when } \\
\text { purchasing groceries or visiting fast } \\
\text { food restaurants, reading and } \\
\text { interpreting food labels } \\
\text { - Followed by monthly } 30-\text { mins } \\
\text { sessions over } 8 \text { weeks } \\
\text { Follow up: } 3 \text { months } \\
\text { Educator: Study coordinator }\end{array}$ & $\begin{array}{l}\text { Receive usual } \\
\text { care from } \\
\text { dietician and } \\
\text { nephrologist } \\
\text { Follow up: } 3 \\
\text { months } \\
\text { Educator: } \\
\text { Study } \\
\text { coordinator }\end{array}$ & $\begin{array}{l}\downarrow \text { sig } \mathrm{PO}^{4} \\
\mathrm{ES}-.41 \\
\uparrow \text { sig Knowledge } \\
\text { ES }-.26\end{array}$ \\
\hline $\begin{array}{l}\text { Karamanidou et } \\
\text { al., } 2008^{43}\end{array}$ & $\begin{array}{l}\text { Study design: } \\
\text { Randomized control } \\
\text { trial }\end{array}$ & $\begin{array}{l}\text { Inclusion criteria: } \\
\text { On HD for at least } 6 \\
\text { months and on } \\
\text { phosphate-binding } \\
\text { medication } \\
\text { Intervention: } \mathbf{N}=19 \\
\text { Mean age } \pm \text { SD: } \\
57.7(14.86) \\
\text { Gender } \mathrm{N}(\%) \\
\text { Male } 10(52.6) \\
\text { Control: } \mathbf{N}=\mathbf{2 0} \\
\text { Mean age } \pm \text { SD: } \\
59.2(16.92) \\
\text { Gender } \mathrm{N}(\%) \\
\text { Male } 10(50.0)\end{array}$ & $\begin{array}{l}\text { Single educational individual } \\
\text { session } \\
\text { over } 16 \text { weeks, length not given } \\
\text {-Patients provided with a leaflet to } \\
\text { improve understanding of the } \\
\text { problem and treatment of high } \\
\text { phosphate } \\
\text { - A demonstration of phosphate } \\
\text { binder medication mode of action } \\
\text { done } \\
\text { Follow up: At weeks } 4 \text { and } 16 \text { post } \\
\text { intervention } \\
\text { Educator: Investigator }\end{array}$ & $\begin{array}{l}\text { Received } \\
\text { usual care } \\
\\
\text { Follow up: At } \\
\text { weeks } 4 \text { and } \\
16 \text { post } \\
\text { intervention } \\
\text { Educator: } \\
\text { Investigator }\end{array}$ & $\begin{array}{l}\downarrow N S P^{4} \\
\text { ES -.11 } \\
\uparrow s i g \text { Adherence } \\
\text { ES } 6.31 \\
\text { Increased knowledge might } \\
\text { have influenced an increase in } \\
\text { adherence behavior }\end{array}$ \\
\hline
\end{tabular}




\begin{tabular}{|c|c|c|c|c|c|}
\hline & & Location: Not given & & & \\
\hline $\begin{array}{l}\text { Sun et al., } \\
2008^{41}\end{array}$ & $\begin{array}{l}\text { Study design: } \\
\text { Before and after } \\
\text { study }\end{array}$ & $\begin{array}{l}\text { Inclusion criteria: } \\
\text { Pre-dialysis } \mathrm{PO}^{4} \text { greater } \\
\text { than } 1.94 \mathrm{mmol} / \mathrm{L}(6.0 \\
\mathrm{mg} / \mathrm{dL}) \\
\text { Intervention: } \mathbf{N}=\mathbf{5 0} \\
\text { Mean age } \\
53.80 \pm 13.98 \\
\text { Gender } \\
\text { Female } 2958 \% \\
\text { Male } 2142 \% \\
\text { Location: Taiwan }\end{array}$ & $\begin{array}{l}30 \text { min individualized education on } \\
\text { how to improve basic knowledge of } \\
\text { hyperphosphatemia and its } \\
\text { complications, intervention period } \\
\text { over } 4 \text { weeks } \\
\text { - patients given a standard booklet } \\
\text { - Education repeated until the } \\
\text { patient passed a post education } \\
\text { evaluation } \\
\text { Follow up: } 12 \text { weeks post } \\
\text { intervention } \\
\text { Educator: Single well-trained } \\
\text { nephrology nurse }\end{array}$ & $\mathbf{N}=\mathbf{0}$ & $\begin{array}{l}\downarrow \text { sig } \mathrm{PO}^{4} \\
\mathrm{ES}-.43 \\
\uparrow \text { sig Knowledge }\end{array}$ \\
\hline $\begin{array}{l}\text { Morey et al., } \\
2008^{47}\end{array}$ & $\begin{array}{l}\text { Study design: } \\
\text { Randomized control } \\
\text { trial }\end{array}$ & $\begin{array}{l}\text { Inclusion criteria: } \\
\text { A mean } \mathrm{PO} 4<1.8 \\
\text { mmol/L }(5.6 \mathrm{mg} / \mathrm{dL}) \text {, on } \\
\text { maintenance } \mathrm{HD}>6 \\
\text { months, age }>18 \text { years, } \\
\text { ability to give informed } \\
\text { consent, a stable urea } \\
\text { reduction rate }>65 \% \text {, } \\
\text { and a body mass index } \\
>20 \\
\text { Intervention: } \mathrm{N}=\mathbf{3 4} \\
\text { Age in years } \mathrm{SD} \text { : } \\
60.4 \pm 15.6 \\
\text { Gender } \\
\text { Women } 26.5 \\
\text { Men } 73.5\end{array}$ & $\begin{array}{l}\text { Individualized monthly education on } \\
\text { dietary phosphate and improving } \\
\text { adherence with phosphate binder } \\
\text { treatment over } 4 \text { weeks. } \\
\text { - All patents were recommended to } \\
\text { visit their regular unit dietician for } \\
\text { standard care if referred. } \\
\text { Follow up: At weeks } 24 \text { post } \\
\text { intervention } \\
\text { Educator: Dietician }\end{array}$ & $\begin{array}{l}\text { Same } \\
\text { intervention as } \\
\text { the } \\
\text { interventions } \\
\text { group with the } \\
\text { exception of } \\
\text { follow up } \\
\text { Follow up: At } \\
\text { weeks } 24 \text { post } \\
\text { intervention } \\
\text { Educator: } \\
\text { Dietician }\end{array}$ & $\begin{array}{l}\downarrow \text { sig } \mathrm{PO}^{4} \\
\mathrm{ES}-.08\end{array}$ \\
\hline
\end{tabular}




\begin{tabular}{|c|c|c|c|c|c|}
\hline & & $\begin{array}{l}\text { Control: } \mathbf{N}=\mathbf{3 3} \\
\text { Age in years SD: } \\
54.9 \pm 51.9 \\
\text { Gender } \\
\text { Woman: } 48.5 \\
\text { Men :1.5 } \\
\text { Location: United } \\
\text { Kingdom }\end{array}$ & & & \\
\hline $\begin{array}{l}\text { Yokum et al., } \\
2008^{48}\end{array}$ & $\begin{array}{l}\text { Study design: } \\
\text { Randomized control } \\
\text { trial }\end{array}$ & $\begin{array}{l}\text { Inclusion criteria: } \\
\text { Age }>18 \text { years, clinically } \\
\text { stable condition, fluency } \\
\text { in English, mental } \\
\text { alertness, and elevated } \\
\mathrm{PO}^{4} \text { level of at least one } \\
\text { value }>1.8 \mathrm{mmol} / \mathrm{L} \text { (5.6 } \\
\text { mg/dL) } \\
\text { Intervention: } \mathrm{N}=17 \\
\text { Age in years SD } \\
51.1 \pm 12.7 \\
\text { Gender } \\
\text { Male: female ratio } \\
11 \mathrm{M}: 6 \mathrm{~F} \\
\text { Control: } \mathrm{N}=17 \\
\text { Age in years SD } \\
47.6 \pm 14.4 \\
\text { Gender } \\
\text { Male: female ratio } \\
12 \mathrm{M}: 5 \mathrm{~F} \\
\text { Location: UK }\end{array}$ & $\begin{array}{l}\text { Monthly individualized consultations } \\
\text { over } 16 \text { weeks on administration } \\
\text { and } \\
\text { adjustment of phosphate binder } \\
\text { dose in relation to meal size } \\
\text { - Phosphate managed according to } \\
\text { a defined protocol decision tree } \\
\text { Follow up: None } \\
\text { Educator: Renal research } \\
\text { pharmacist and renal research } \\
\text { dietician }\end{array}$ & $\begin{array}{l}\text { Renal } \\
\text { research } \\
\text { dietician } \\
\text { provided } \\
\text { counselling but } \\
\text { no pharmacist } \\
\text { consult } \\
\text { Follow up: } \\
\text { None } \\
\text { Educator: } \\
\text { Renal } \\
\text { research } \\
\text { pharmacist } \\
\text { and renal } \\
\text { research } \\
\text { dietician }\end{array}$ & $\begin{array}{l}\downarrow \text { sig } \mathrm{PO}^{4} \\
\mathrm{ES}-2.69\end{array}$ \\
\hline
\end{tabular}

$\mathrm{PO}^{4}$ : Serum phosphate given as $\mathrm{mmol} / \mathrm{L}$ 


\section{Appendix V: Excluded studies}

Brogdon, R.M. A self-care educational intervention to improve knowledge of dietary phosphorus control in patients requiring hemodialysis: A pilot study

Reason for exclusion: Methodology lacked rigor, inclusion criteria for inclusion in study not defined.

Chow, J. \& Dalton, B. A novel, 12-week, nurse-led motivation and education programme has no effect on serum phosphate levels in haemodialysis patient.

Reason for exclusion: Methodology lacked rigor, it scored low quality across several criteria on the JBI critical checklist for Descriptive/Case

Righetto de Araujo1, L., Figueiredo, a., Lorenzoni d'Avil., D. Evaluation of an educational program on calcium and phosphorus metabolism for patients on hemodialysis.

Reason for exclusion: Methodology lacked rigor, it scored unclear across several criteria on the JBI critical checklist for Descriptive/Case

Baraz S.H., Parvardeh S., Mohammadi E. \& Broumand B. Dietary and fluid compliance: an educational intervention for patients having haemodialysis.

Reason for exclusion: This study lacked specific education on phosphate control

Eff, D \& Kocaöz S. Adherence to diet and fluid restriction of individuals on hemodialysis treatment and affecting factors in Turkey

Reason for exclusion: This study lacked specific education on phosphate control

Karavetian, M and Ghaddar, S. Nutritional education for the management of osteodystrophy (nemo) in patients on haemodialysis

Reason for exclusion: No outcomes reported, paper reported only the study design

Ogna VF, Pruijm M, Zweiacker C, Wuerzner G, Tousset E, and Burnier M. Clinical benefits of an adherence monitoring program in the management of secondary hyperparathyroidism with cinacalcet

Reason for exclusion: Study did not focused on specific phosphate control and did not report serum phosphate results

Reese P, Mgbako O, Mussel I A, Potluri V, Yekta Z, Levsky S, Bellamy S, Parikh C, Shults J, Glanz $\mathrm{K}$,Feldman $\mathrm{H}$, Volpp K. A pilot randomized trial of financial incentives or coaching to lower serum phosphorus in dialysis patients

Reason for exclusion: Study results for serum phosphate reported as interquartile range not as standard deviation 
San Miguel, S., Curtale, M, Knagge, D, Nhan, C \& Chow, J. Improving patient understanding of phosphate binders: A bony challenge.

Reason for exclusion: This study included pre HD and peritoneal dialysis patient and outcomes of interest not clearly defined

Sevick, M., Stone, R., Novak, M., Piraino, B., Snetselaar, L., Marsh, R., Hall, B., Lash, H., Bernardini, J., Burke, L. A PDA-based dietary self-monitoring intervention to reduce sodium intake in an in-centre hemodialysis patient.

Reason for exclusion: Study did not focused on specific phosphate control and had a small sample size

Umeukeje, E. M., Merighi J., Browne T., VictorofF J.N., Umanath K., Lewis J.B., Ikizler A. T., Wallston K.A., Cavanaugh K. Self-motivation is associated with phosphorus control in end-stage renal disease.

Reason for exclusion: This study lacked specific education on phosphate control 


\section{Appendix VI}

Forest plot of seven experimental studies on effects of educational or behavioral intervention compared with standard care on serum phosphate levels

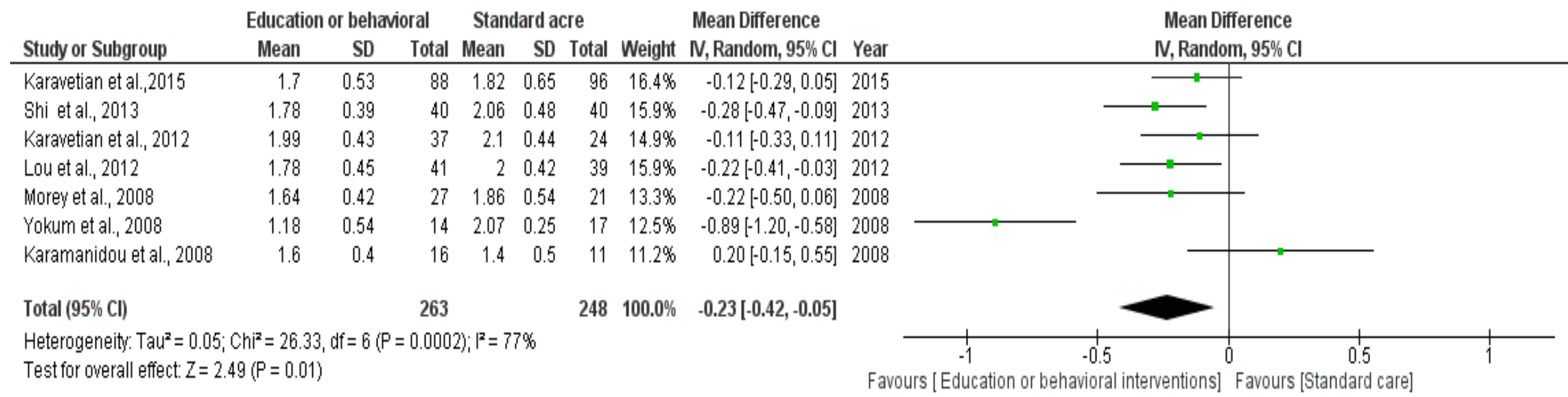


JBI Database of Systematic Reviews \& Implementation Reports 